\title{
ASYMPTOTIC BEHAVIOR OF SECOND-ORDER DISSIPATIVE EVOLUTION EQUATIONS COMBINING POTENTIAL WITH NON-POTENTIAL EFFECTS*
}

\author{
Hedy Attouch ${ }^{1}$ And Paul-Émile Maingé ${ }^{2}$
}

\begin{abstract}
In the setting of a real Hilbert space $\mathcal{H}$, we investigate the asymptotic behavior, as time $t$ goes to infinity, of trajectories of second-order evolution equations

$$
\ddot{u}(t)+\gamma \dot{u}(t)+\nabla \phi(u(t))+A(u(t))=0,
$$

where $\nabla \phi$ is the gradient operator of a convex differentiable potential function $\phi: \mathcal{H} \rightarrow \mathbb{R}, A: \mathcal{H} \rightarrow \mathcal{H}$ is a maximal monotone operator which is assumed to be $\lambda$-cocoercive, and $\gamma>0$ is a damping parameter. Potential and non-potential effects are associated respectively to $\nabla \phi$ and $A$. Under condition $\lambda \gamma^{2}>1$, it is proved that each trajectory asymptotically weakly converges to a zero of $\nabla \phi+A$. This condition, which only involves the non-potential operator and the damping parameter, is sharp and consistent with time rescaling. Passing from weak to strong convergence of the trajectories is obtained by introducing an asymptotically vanishing Tikhonov-like regularizing term. As special cases, we recover the asymptotic analysis of the heavy ball with friction dynamic attached to a convex potential, the second-order gradient-projection dynamic, and the second-order dynamic governed by the Yosida approximation of a general maximal monotone operator. The breadth and flexibility of the proposed framework is illustrated through applications in the areas of constrained optimization, dynamical approach to Nash equilibria for noncooperative games, and asymptotic stabilization in the case of a continuum of equilibria.
\end{abstract}

Mathematics Subject Classification. 34C35, 34D05, 65C25, 90C25, 90C30.

Received May 1st, 2009. Revised December 18, 2009 and April 9, 2010.

Published online August 6, 2010.

\section{INTRODUCTION}

Throughout this paper, $\mathcal{H}$ is a real Hilbert space. Its scalar product is denoted by $\langle\cdot, \cdot\rangle$ and the associated norm by $|\cdot|$. We wish to investigate the asymptotic behavior, as the time variable $t$ goes to $+\infty$, of second

\footnotetext{
Keywords and phrases. Second-order evolution equations, asymptotic behavior, dissipative systems, maximal monotone operators, potential and non-potential operators, cocoercive operators, Tikhonov regularization, heavy ball with friction dynamical system, constrained optimization, coupled systems, dynamical games, Nash equilibria.

* Dedicated to Alain Haraux on the occasion of his 60th birthday.

${ }^{1}$ Institut de Mathématiques et de Modélisation de Montpellier, UMR CNRS 5149, CC 51, Université Montpellier II, place Eugène Bataillon, 34095 Montpellier Cedex 5, France. attouch@math.univ-montp2.fr.

Supported by French ANR grant ANR-08-BLAN-0294-03.

2 Université des Antilles-Guyane, D.S.I., CEREGMIA, Campus de Schoelcher, 97233 Schoelcher Cedex, Martinique, France.

Paul-Emile.Mainge@martinique.univ-ag.fr
} 
order evolution equations

$$
\ddot{u}(t)+\gamma \dot{u}(t)+\mathcal{A}(u(t))=0
$$

where $\mathcal{A}: \mathcal{H} \rightarrow \mathcal{H}$ is a maximal monotone operator and $\gamma>0$ is a positive damping parameter. Here and henceforth, a $\operatorname{dot}\left({ }^{*}\right)$ denotes first-order differentiation with respect to time $t$, and a double dot $\left({ }^{*}\right)$ denotes second-order differentiation. In order to grasp the respective influence of potential and non-potential operators in such inertial systems, and their effects on the asymptotic behavior of trajectories, we consider the class of maximal monotone operators which can be splitted up into the sum of two terms $\mathcal{A}=\nabla \phi+A$ where

- $\nabla \phi: \mathcal{H} \rightarrow \mathcal{H}$ is the gradient operator of a convex and continuously differentiable function $\phi: \mathcal{H} \rightarrow \mathbb{R}$

- $A: \mathcal{H} \rightarrow \mathcal{H}$ is a maximal monotone operator that is assumed to be cocoercive, which means that there exists some constant $\lambda>0$ such that

$$
\forall(v, w) \in \mathcal{H}^{2}\langle A v-A w, v-w\rangle \geq \lambda|A v-A w|^{2} .
$$

An operator $A: \mathcal{H} \rightarrow \mathcal{H}$ which satisfies (1.2) is said to be $\lambda$-cocoercive (the relevancy of this hypothesis with respect to applications is examined below). Note that $A \lambda$-cocoercive implies that $A$ is $(1 / \lambda)$-Lipschitz continuous.

An outline of the present work is as follows:

1. In Section 2, we study the asymptotic behavior of second-order autonomous evolution systems governed by such operators $\mathcal{A}=\nabla \phi+A$, namely

$$
\ddot{u}(t)+\gamma \dot{u}(t)+\nabla \phi(u(t))+A(u(t))=0,
$$

with $\phi$ convex and continuously differentiable, $A \lambda$-cocoercive for some $\lambda>0$, and $\gamma>0$ as a damping parameter. Let us denote by

$$
S:=\{v \in \mathcal{H} \quad \mid \nabla \phi(v)+A v=0\}
$$

the set of equilibria and suppose that $S \neq \emptyset$. In Theorem 2.1 we establish that, under the sole assumption

$$
\lambda \gamma^{2}>1
$$

each trajectory $t \rightarrow u(t)$ of (1.3) weakly converges in $\mathcal{H}$, as $t \rightarrow+\infty$, to an element of $S$. This condition, which only involves the non-potential part of the maximal monotone operator governing the equation, is proved to be sharp, in the sense that one can exhibit situations where condition $\lambda \gamma^{2}<1$ does not ensure convergence of all trajectories.

When the operator $\nabla \phi+A$ is strongly monotone we prove strong asymptotic convergence of the trajectories.

In the potential case, $\mathcal{A}=\nabla \phi$ (corresponding to $A=0$ ), taking advantage of the fact that, in the above result, no restrictive assumption is made on the potential operator $\nabla \phi$, we recover the asymptotic convergence result for the so-called heavy ball with friction dynamical system (Alvarez [2])

$$
\ddot{u}(t)+\gamma \dot{u}(t)+\nabla \phi(u(t))=0 .
$$

In recent years, because of its rich connections with mechanics and optimization, this system has been the object of active research, see $[2,8,13,14]$, and the references herein.

In the non-potential case, $\nabla \phi=0,(1.3)$ becomes

$$
\ddot{u}(t)+\gamma \dot{u}(t)+A(u(t))=0
$$

with $A$ a cocoercive operator. Equation (1.7) covers several situations of practical interest:

- $A=I-T$ where $T: \mathcal{H} \rightarrow \mathcal{H}$ is a contraction. It can be easily checked that $A$ is $(1 / 2)$-cocoercive. Condition (1.5) gives $\gamma>\sqrt{2}$. When specialized to this situation, Theorem 2.1 yields convergence results for the second order gradient-projection dynamical system, first established in $[4,8]$. 
- $A=B_{\lambda}$ where $B_{\lambda}$ (with parameter $\lambda>0$ ) is the Yosida approximation of a general maximal monotone operator $B: \mathcal{H} \rightarrow 2^{\mathcal{H}}$ (see [20]). One can easily verify that $B_{\lambda}$ is $\lambda$-cocoercive. Noticing that $B$ and $B_{\lambda}$ have the same zeroes, we shall derive new inertial second-order dynamical approach to the set of zeroes $B^{-1}(0)$, for $B$ a general maximal monotone operator.

2. In Section 3, we introduce a Tikhonov-like regularizing term $\nabla \Theta(u(t))$ with vanishing coefficient $\epsilon(t)$ in the above dynamic, and consider the nonautonomous system

$$
\ddot{u}(t)+\gamma \dot{u}(t)+\nabla \phi(u(t))+A(u(t))+\epsilon(t) \nabla \Theta(u(t))=0,
$$

where the function $\Theta: \mathcal{H} \rightarrow \mathbb{R}$ is supposed to be convex, differentiable, with $\nabla \Theta$ Lipschitz continuous and strongly monotone, while $\epsilon:[0,+\infty) \rightarrow[0,+\infty)$ is a function of class $C^{1}$ such that $\epsilon(t) \rightarrow 0^{+}$as $t \rightarrow+\infty$.

In Theorem 3.1, we establish that, under condition (1.5), and the slow vanishing condition on $\epsilon(\cdot)$

$$
\int_{0}^{+\infty} \epsilon(s) \mathrm{d} s=+\infty,
$$

each trajectory of (1.8) strongly converges as $t \rightarrow+\infty$ to $u_{*}$, which is the unique minimizer of $\Theta$ over the set $S:=(\nabla \phi+A)^{-1}(0)$. Equivalently, $u_{*}$ solves the following variational inequality problem: find $u_{*} \in S$ such that

$$
\left\langle\nabla \Theta\left(u_{*}\right), v-u_{*}\right\rangle \geq 0 \quad \forall v \in S .
$$

Note that the above regularization technique allows both to obtain strong convergence of the trajectories, together with a limit which no longer depends on the initial data. As a consequence of Theorem 3.1, we recover various results which have been devoted to the Tikhonov dynamics, see [10,24] and the references therein.

3. In the final section, we briefly outline situations where our results can be applied. Indeed, the convergence results obtained in Theorems 2.1 and 3.1 offer promising views on numerical optimization and algorithms (by time discretization as in $[2,5,9,23])$, on the modeling of inertial dynamical approach to Nash equilibria in decision sciences and game theory (see [11,24]), and on dissipative dynamical systems and PDE's (as in [6] for the damped wave equation).

\section{Asymptotic COnVERGence Results}

This section is devoted to the study of the asymptotic behavior, as time variable $t$ goes to infinity, of trajectories of (1.3).

\subsection{Weak asymptotic convergence results}

Throughout this section we make the following assumptions:

(H1) $\phi: \mathcal{H} \rightarrow \mathbb{R}$ is a convex differentiable function whose gradient $\nabla \phi$ is Lipschitz continuous on the bounded subsets of $\mathcal{H}$;

(H2) $A: \mathcal{H} \rightarrow \mathcal{H}$ is maximal monotone and $\lambda$-cocoercive for some $\lambda>0$.

Note that the cocoerciveness of $A$ implies that $A$ is Lipschitz continuous, so that $\nabla \phi+A$ is Lipschitz continuous on bounded sets. By Cauchy-Lipschitz theorem, for any initial data $u(0)=u_{0}, \dot{u}(0)=v_{0}$ with $\left(u_{0}, v_{0}\right)$ in $\mathcal{H}^{2}$ there exists a unique local solution to the Cauchy problem

$$
\left[\begin{array}{l}
\ddot{u}(t)+\gamma \dot{u}(t)+\nabla \phi(u(t))+A(u(t))=0, \\
u(0)=u_{0}, \quad \dot{u}(0)=v_{0} .
\end{array}\right.
$$

The fact that $u$ is infinitely extendible to the right follows from a uniform bound on $|\dot{u}(t)|$ as given in the proof of Theorem 2.1, which provides a unique classical global solution $u \in C^{2}([0,+\infty) ; \mathcal{H})$ of $(2.1)$.

The following theorem establishes the weak asymptotic convergence property of $u(\cdot)$ solution of $(2.1)$, under the sole assumption $\lambda \gamma^{2}>1$. 
Theorem 2.1. Let us suppose that (H1)-(H2) hold with $S:=(\nabla \phi+A)^{-1}(0) \neq \emptyset$, and that the cocoercive parameter $\lambda$ and the damping parameter $\gamma$ satisfy

$$
\lambda \gamma^{2}>1
$$

Then, for each initial data $u_{0}$ and $v_{0}$ in $\mathcal{H}$, the unique solution $u \in C^{2}([0,+\infty) ; \mathcal{H})$ of $(2.1)$ satisfies:

(i1) There exists $u_{\infty} \in S$ such that $u(t) \rightarrow u_{\infty}$ weakly in $\mathcal{H}$ as $t \rightarrow+\infty$.

Moreover,

(i2) $\dot{u} \in L^{2}(0,+\infty ; \mathcal{H}) ; \lim _{t \rightarrow+\infty}|\dot{u}(t)|=0$;

(i3) $\ddot{u}+\nabla \phi(u)+A p \in L^{2}(0,+\infty ; \mathcal{H})$ whenever $p \in S$;

(i4) for every $p \in S$, $\lim _{t \rightarrow+\infty}|u(t)-p|$ exists.

Before proceeding with the proof of Theorem 2.1, we establish three technical lemmas. The following lemma plays a crucial role in the proof of optimality of the weak cluster points of a given trajectory of (2.1). Because of its independent interest, it is stated in a general setting, the potential $\Phi$ being allowed to be non-smooth. We use the classical notation $\partial \Phi$ for the subdifferential operator of $\Phi$ (it coincides with the gradient operator in the smooth case). This lemma can be viewed as a generalization of Lemma 1 in [17].

Lemma 2.1. Let $\Phi: \mathcal{H} \rightarrow \mathbb{R} \cup\{+\infty\}$ be a convex and lower semicontinuous function, $\left(u_{n}\right)$ a bounded sequence in $\mathcal{H}$, and $p \in \mathcal{H}$. If there exist $\eta_{n} \in \partial \Phi\left(u_{n}\right)$ and $\bar{\eta} \in \partial \Phi(p)$ such that

$$
\lim _{n \rightarrow+\infty}\left\langle u_{n}-p, \eta_{n}-\bar{\eta}\right\rangle=0,
$$

then any weak cluster point $\bar{u}$ of $\left(u_{n}\right)$ satisfies

$$
\bar{\eta} \in \partial \Phi(\bar{u}) .
$$

Proof. Set $w_{n}=\left\langle u_{n}-p, \eta_{n}-\bar{\eta}\right\rangle$ and introduce the functional $F: \mathcal{H} \rightarrow \mathbb{R} \cup\{+\infty\}$ defined for any $v \in \mathcal{H}$ by

$$
F(v)=\Phi(v)-\Phi(p)-\langle v-p, \bar{\eta}\rangle .
$$

By convexity of $\Phi$ and $\bar{\eta} \in \partial \Phi(p)$, we observe that $F$ is a convex and nonnegative function, and it can be easily checked that

$$
F\left(u_{n}\right)=\Phi\left(u_{n}\right)-\Phi(p)-\left\langle u_{n}-p, \eta_{n}\right\rangle+w_{n} .
$$

Therefore, from convexity of $\Phi$ and $\eta_{n} \in \partial \Phi\left(u_{n}\right)$, we have

$$
0 \leq F\left(u_{n}\right) \leq w_{n},
$$

which by (2.3) (that is $w_{n} \rightarrow 0$ as $n \rightarrow+\infty$ ) gives

$$
\lim _{n \rightarrow+\infty} F\left(u_{n}\right)=0 .
$$

Now consider any weak cluster point $\bar{u}$ of $\left(u_{n}\right)$, namely there exists a subsequence $\left(u_{n_{k}}\right)$ of $\left(u_{n}\right)$ such that $\left(u_{n_{k}}\right)$ weakly converges to $\bar{u}$ as $k \rightarrow+\infty$. Then invoking the weak lower semicontinuity of $F$ (as it is convex and lower semicontinuous) and using (2.6), we obtain

$$
0 \leq F(\bar{u}) \leq \liminf _{k \rightarrow+\infty} F\left(u_{n_{k}}\right)=\lim _{n \rightarrow+\infty} F\left(u_{n}\right)=0,
$$

which entails $F(\bar{u})=0$. This implies that $\bar{u}$ is a minimizer of the convex and nonnegative function $F$, so that $0 \in \partial F(\bar{u})$, which from $(2.5)$ is equivalent to $(2.4)$. 
Remark 2.1. Lemma 2.1 still holds true in the more general situation, where instead of a subdifferential operator $\partial \Phi$, one considers a maximal monotone operator which is angle-bounded, see [16] for the definition and study of this class of operators. Indeed, the conclusion of Lemma 2.1 can be obtained as a straight consequence of the angle-bounded property.

Lemma 2.2. Let $B: \mathcal{H} \rightarrow \mathcal{H}$ be a maximal monotone single-valued operator and $\Phi: \mathcal{H} \rightarrow \mathbb{R}$ a convex differentiable function such that $\Omega:=(\nabla \Phi+B)^{-1}(0) \neq \emptyset$. Suppose that $p \in \Omega$ and $\left(u_{n}\right)$ is a bounded sequence in $\mathcal{H}$ verifying

(c1) $\lim _{n \rightarrow+\infty}\left\langle\nabla \Phi\left(u_{n}\right)-\nabla \Phi(p), u_{n}-p\right\rangle=0$,

(c2) $\lim _{n \rightarrow+\infty}\left|B u_{n}-B p\right|=0$.

Then, any weak cluster point of $\left(u_{n}\right)$ belongs to $\Omega$.

Proof. Let $\bar{u}$ be a weak cluster point of $\left(u_{n}\right)$. From (c1) and invoking Lemma 2.1, we have $\nabla \Phi(p)=\nabla \Phi(\bar{u})$. Moreover, from (c2) and recalling that the maximal monotone operator $B$ has a graph which is closed in $w-\mathcal{H} \times s-\mathcal{H}$ (see [20] for example), we have $B \bar{u}=B p$. As a straightforward consequence, $\nabla \Phi(\bar{u})+B \bar{u}=$ $\nabla \Phi(p)+B p=0$, so that $\bar{u} \in \Omega$, which completes the proof.

Remark 2.2. Note that the proof of Lemma 2.2 is based on the general Lemma 2.1, even though our study is only concerned with the case when $\nabla \Phi$ is Lipschitz continuous on bounded sets. It is also clear that Lemma 2.2 could have been stated independently from Lemma 2.1 in some situation when $\nabla \Phi$ is cocoercive on bounded sets. However, the Baillon-Haddad theorem [16] (which state the equivalence between Lipschitz continuity and cocoerciveness of $\nabla \Phi)$ does not seem to be adapted to get such a situation because it is not locally applicable.

The following lemma will be used in the proof of Theorem 2.1 in order to obtain the asymptotic convergence of the mapping $t \rightarrow|u(t)-p|$, whenever $p \in S$ and $u$ is solution of (2.1). This lemma appears implicitly in [2], its proof is given for the sake of completeness.

Lemma 2.3. If $w \in C^{2}([0,+\infty) ; \mathbb{R})$ is bounded from below and satisfies the following inequality

$$
\ddot{w}(t)+\gamma \dot{w}(t) \leq g(t)
$$

where $\gamma$ is a positive constant and $g \in L^{1}([0,+\infty) ; \mathbb{R})$, then $w(t)$ converges as $t \rightarrow+\infty$.

Proof. From inequality $(2.7)$ and denoting $[\dot{w}]_{+}=\max \{\dot{w}(t), 0\}$, we classically have $[\dot{w}(t)]_{+} \leq \mathrm{e}^{-\gamma t}[\dot{w}(0)]_{+}+$ $\int_{0}^{t} \mathrm{e}^{-\gamma(t-\tau)}|g(\tau)| \mathrm{d} \tau$, while Fubini's theorem gives us $\int_{0}^{+\infty} \int_{0}^{t} \mathrm{e}^{-\gamma(t-\tau)}|g(\tau)| \mathrm{d} \tau \mathrm{d} t=\frac{1}{\gamma} \int_{0}^{+\infty}|g(\tau)| \mathrm{d} \tau<+\infty$. This shows that $[\dot{w}]_{+} \in L^{1}([0,+\infty) ; \mathbb{R})$. Now setting $z(t)=w(t)-\int_{0}^{t}[\dot{w}(\tau)]_{+} \mathrm{d} \tau$, we observe that $z($.$) is bounded$ from below (as $w($.$) is assumed to be bounded from below) with \dot{z}(t)=\dot{w}(t)-[\dot{w}(t)]_{+} \leq 0$, hence $z(t)$ converges as $t \rightarrow+\infty$, and so does $w(t)$.

The following remarks aim at making the proof of Theorem 2.1 the more legible as possible.

Remark 2.3. It is a simple matter to check that, for any elements $p, q$ in $\mathcal{H}$ such that $\nabla \phi(p)+A p=\nabla \phi(q)+$ $A q$, we have $\nabla \phi(p)=\nabla \phi(q)$ and $A p=A q$ (since $A$ is assumed to be cocoercive). As a consequence, for $p \in S, \nabla \phi(p)$ and $A p$ are completely determined. Thus there exists a unique element $d \in \mathcal{H}$ such that $S=$ $\{p \in \mathcal{H}, \quad A p=d, \quad \nabla \phi(p)=-d\}$. Considering the function $\Phi: \mathcal{H} \rightarrow \mathbb{R}$ and the operator $B: \mathcal{H} \rightarrow \mathcal{H}$ defined by

$$
\Phi=\phi+\langle d, .\rangle \text { and } B=A-d,
$$

we observe that $S$ can be reduced to

$$
S=\{p \in \mathcal{H}, \quad \nabla \Phi(p)=0, \quad B p=0\} .
$$

Moreover, $B$ is obviously checked to be $\lambda$-cocoercive, as $A$ is assumed to satisfy this latter property. 
Remark 2.4. Clearly, the solution $u$ of (2.1) satisfies $\ddot{u}+\gamma \dot{u}+\nabla \Phi(u)+B u=0$, where $\Phi$ and $B$ are given by (2.8). Given $p \in S$ and setting $v=u-p$, we then immediately observe that $v$ is solution of

$$
\ddot{v}+\gamma \dot{v}+\nabla \bar{\phi}(v)+\bar{A} v=0,
$$

where $\bar{A} v=B(v+p)$ and $\bar{\phi}(v)=\Phi(v+p)-\Phi(p)$. It is easily seen that $\bar{A}$ is $\lambda$-cocoercive (as it is for $B$, by condition (H2)), $\bar{\phi}$ is convex and its gradient is Lipschitz continuous on the bounded subsets of $\mathcal{H}$ (by (H1)). Furthermore, in light of Remark 2.3, we have

$$
\nabla \bar{\phi}(0)=\nabla \phi(p)+d=0 \text { and } \bar{A} 0=A p-d=0 .
$$

Remark 2.5. As a straightforward consequence of Lemma 2.2, we obtain that if $\left(v_{n}\right)$ is a bounded sequence in $\mathcal{H}$ such that

$$
\lim _{n \rightarrow+\infty}\left\langle\nabla \bar{\phi}\left(v_{n}\right), v_{n}\right\rangle=0 \text { and } \lim _{n \rightarrow+\infty}\left|\bar{A} v_{n}\right|=0
$$

then any weak cluster point of $\left(v_{n}+p\right)$ belongs to $S$ ( $p$ being the element of $S$ involved in the definition of $\bar{\phi}$ and $\bar{A})$. Indeed, setting $u_{n}=v_{n}+p$, we have $\left\langle\nabla \bar{\phi}\left(v_{n}\right), v_{n}\right\rangle=\left\langle\nabla \Phi\left(u_{n}\right), u_{n}-p\right\rangle$ and $\left|\bar{A} v_{n}\right|=\left|B u_{n}\right|$. As $\nabla \Phi(p)=0$ and $B p=0$ (according to the alternative characterization of $S$ given by (2.9)), we can therefore apply Lemma 2.2 to get the desired result.

We are now in position to prove the main result of this section.

Proof of Theorem 2.1. We begin with the proof of items (i2)-(i4). To that end, we equivalently establish that the solution $v$ of (2.10) satisfies: $\dot{v}$ and $\ddot{v}+\nabla \bar{\phi}(v)$ belong to $L^{2}(0,+\infty ; \mathcal{H}),|\dot{v}(t)| \rightarrow 0$ and $|v(t)|$ converges as $t \rightarrow+\infty$. Set $h(t)=(1 / 2)|v(t)|^{2}$. From $\dot{h}(t)=\langle v(t), \dot{v}(t)\rangle$ and $\ddot{h}(t)=\langle v(t), \ddot{v}(t)\rangle+|\dot{v}(t)|^{2}$ we obtain

$$
\ddot{h}(t)+\gamma \dot{h}(t)=\langle v(t), \ddot{v}(t)+\gamma \dot{v}(t)\rangle+|\dot{v}(t)|^{2},
$$

which, by using (2.10) yields

$$
\ddot{h}(t)+\gamma \dot{h}(t)+\langle\nabla \bar{\phi}(v(t)), v(t)\rangle+\langle\bar{A} v(t), v(t)\rangle=|\dot{v}(t)|^{2} .
$$

Then, by $\nabla \bar{\phi}(0)=0, \bar{A} 0=0$, and using the $\lambda$-cocoerciveness of $\bar{A}$, together with the monotonicity of $\nabla \bar{\phi}$ (since $\bar{\phi}$ is convex), we obtain

$$
\ddot{h}(t)+\gamma \dot{h}(t)+\lambda|\bar{A} v(t)|^{2} \leq|\dot{v}(t)|^{2} .
$$

Regarding the third term in the left side of (2.15), by using (2.10) again, replacing $\bar{A} v(t)$ by $-(\ddot{v}(t)+\gamma \dot{v}(t)+$ $\nabla \bar{\phi}(v(t)))$, and setting $D(t):=|\ddot{v}(t)+\nabla \bar{\phi}(v(t))|^{2}$, we obtain

$$
\begin{aligned}
|\bar{A} v(t)|^{2} & =|(\ddot{v}(t)+\nabla \bar{\phi}(v(t)))+\gamma \dot{v}(t)|^{2} \\
& =D(t)+\gamma^{2}|\dot{v}(t)|^{2}+2 \gamma\langle\dot{v}(t), \ddot{v}(t)+\nabla \bar{\phi}(v(t))\rangle \\
& =D(t)+\gamma^{2}|\dot{v}(t)|^{2}+\gamma \frac{\mathrm{d}}{\mathrm{d} t}\left(|\dot{v}(t)|^{2}+2 \bar{\phi}(v(t))\right) .
\end{aligned}
$$

Using the above equality, let us rewrite $(2.15)$ as

$$
\ddot{h}(t)+\gamma \dot{h}(t)+\left(\lambda \gamma^{2}-1\right)|\dot{v}(t)|^{2}+\lambda D(t)+\lambda \gamma \frac{\mathrm{d}}{\mathrm{d} t}\left(|\dot{v}(t)|^{2}+2 \bar{\phi}(v(t))\right) \leq 0 .
$$

By using assumption $\lambda \gamma^{2} \geq 1$, with $D(t) \geq 0$ and (2.16), we obtain

$$
\ddot{h}(t)+\gamma \dot{h}(t)+\lambda \gamma \frac{\mathrm{d}}{\mathrm{d} t}\left(|\dot{v}(t)|^{2}+2 \bar{\phi}(v(t))\right) \leq 0,
$$


which expresses that the function

$$
\Gamma_{0}(t):=\dot{h}(t)+\gamma h(t)+\lambda \gamma\left(|\dot{v}(t)|^{2}+2 \bar{\phi}(v(t))\right)
$$

is nonincreasing on $[0,+\infty)$. Indeed, $\Gamma_{0}(\cdot)$ will serve us as a Lyapunov function in the asymptotic analysis of (2.1). Let us first show the boundedness of trajectories. By convexity of $\bar{\phi}$ and $\nabla \bar{\phi}(0)=0, \bar{\phi}(0)=0$ we obviously have

$$
0 \leq \bar{\phi}(v(t)) .
$$

Combining the nonincreasing property of $\Gamma_{0}(\cdot)$ with the above property we obtain

$$
\dot{h}(t)+\gamma h(t)+\lambda \gamma|\dot{v}(t)|^{2} \leq \Gamma_{0}(t) \leq \Gamma_{0}(0) .
$$

Set

which clearly satisfies

$$
C_{0}:=|v(0)||\dot{v}(0)|+\frac{\gamma}{2}|v(0)|^{2}+\lambda \gamma|\dot{v}(0)|^{2}+2 \lambda \gamma \bar{\phi}(v(0)),
$$

$$
C_{0} \geq \Gamma_{0}(0)
$$

From (2.20) and (2.21) we deduce that $\dot{h}(t)+\gamma h(t) \leq C_{0}$, so that, after integration, we obtain

$$
h(t)=\frac{1}{2}|v(t)|^{2} \leq \frac{1}{2}|v(0)|^{2}+\frac{C_{0}}{\gamma},
$$

which shows that the trajectory $t \rightarrow v(t)$ remains bounded on $[0,+\infty)$ :

$$
\sup _{t \in[0,+\infty)}|v(t)|<+\infty .
$$

Let us now establish estimates on $\dot{v}$ and $\ddot{v}$. Integrating (2.16) from 0 to $t$, together with (2.19) yields

$$
\dot{h}(t)+\gamma h(t)+\lambda \gamma|\dot{v}(t)|^{2}+\left(\lambda \gamma^{2}-1\right) \int_{0}^{t}|\dot{v}(s)|^{2} \mathrm{~d} s+\lambda \int_{0}^{t}|\ddot{v}(s)+\nabla \bar{\phi}(v(s))|^{2} \mathrm{~d} s \leq C_{0},
$$

which implies $\dot{h}(t)+\lambda \gamma|\dot{v}(t)|^{2} \leq C_{0}$. Equivalently, by definition of $h(t)$, we have

$$
\langle v(t), \dot{v}(t)\rangle+\lambda \gamma|\dot{v}(t)|^{2} \leq C_{0}
$$

Since $v(t)$ remains bounded on $[0,+\infty)$, inequality $(2.24)$ implies that $\dot{v}(t)$ also remains bounded on $[0,+\infty)$ :

$$
\sup _{t \in[0,+\infty)}|\dot{v}(t)|<+\infty
$$

From (2.22), (2.25) and $\dot{h}(t)=\langle v(t), \dot{v}(t)\rangle$, we observe that $\dot{h}(t)$ is bounded on $[0,+\infty)$. Returning to (2.23) we deduce that

$$
\int_{0}^{+\infty}|\dot{v}(s)|^{2} \mathrm{~d} s<+\infty \text { and } \int_{0}^{+\infty}|\ddot{v}(s)+\nabla \bar{\phi}(v(s))|^{2} \mathrm{~d} s<+\infty .
$$

Using that $v$ and $\dot{v}$ are bounded on $[0,+\infty)$, together with the Lipschitz continuity of $\bar{A}$ and equation (2.10), we deduce that $\ddot{v}$ is also bounded:

$$
\sup _{t \in[0,+\infty)}|\ddot{v}(t)|<+\infty .
$$

Properties (2.26) and (2.27) express that the function $g:=\dot{v}$ satisfies both

$$
g \in L^{2}(0,+\infty ; \mathcal{H}) \text { and } \dot{g} \in L^{\infty}(0,+\infty ; \mathcal{H}) .
$$


By a classical result, this implies $\lim _{t \rightarrow+\infty} g(t)=0$, that is,

$$
\lim _{t \rightarrow+\infty}|\dot{v}(t)|=0
$$

It remains to prove that $|v(t)|$ converges as $t \rightarrow+\infty$. From (2.14) and the monotonicity of $\bar{A}$, together with $\bar{A} 0=0$, we have

$$
\ddot{h}(t)+\gamma \dot{h}(t)+w(t) \leq|\dot{v}(t)|^{2}
$$

where $w(t)=\langle\nabla \bar{\phi}(v(t)), v(t)\rangle$. It is obviously seen that $w(t)$ is a nonnegative term (thanks to the monotonicity of $\nabla \bar{\phi}$ and $\nabla \bar{\phi}(0)=0)$, hence, from $(2.29)$, we immediately derive

$$
\ddot{h}(t)+\gamma \dot{h}(t) \leq|\dot{v}(t)|^{2} .
$$

Moreover, by $(2.26)$ we know that $|\dot{v}|^{2}$ belongs to $L^{1}(0,+\infty ; \mathcal{H})$. Therefore, noticing that $h$ is nonnegative, Lemma 2.3 shows that

$$
\lim _{t \rightarrow+\infty}|v(t)| \text { exists. }
$$

Now we focus on the proof of item (i1). We have all the ingredients to conclude thanks to Opial's lemma, see [33]. To that end, we need to prove that properties (a) and (b) are fulfilled:

(a) for every $p \in S, \lim _{t \rightarrow+\infty}|u(t)-p|$ exists;

(b) for every $t_{n} \rightarrow+\infty$ with $u\left(t_{n}\right) \rightarrow \bar{u}$ weakly in $\mathcal{H}$, we have $\bar{u} \in S$.

Opial's lemma asserts that, under the above two properties, $u(t)$ weakly converges as $t \rightarrow+\infty$ to an element $u_{\infty} \in S$.

Property (a) is obviously deduced from (2.31).

Let us now prove (b). Returning to (2.10), by using that functions $v$ and $\dot{v}$ are Lipschitz continuous on $[0,+\infty)$ (as their derivatives are uniformly bounded), that $\bar{A}$ is Lipschitz continuous and $\nabla \bar{\phi}$ is Lipschitz continuous on bounded sets, we deduce that $\ddot{v}$ is Lipschitz continuous on $[0,+\infty)$. Once again, this property together with (2.26) implies that $\lim _{t \rightarrow+\infty}|\ddot{v}(t)+\nabla \bar{\phi}(v(t))|=0$, which in light of (2.10) and (2.28) leads to $\lim _{t \rightarrow+\infty}|\bar{A} v(t)|=0$. On the other hand, by (2.29), after integration we obtain $\int_{0}^{+\infty} w(s) \mathrm{d} s<+\infty$. Owing to the Lipschitz continuity property of $w(\cdot)$ we deduce that $\lim _{t \rightarrow+\infty} w(t)=0$. We can now apply Lemma 2.2 (see Rem. 2.5) to finally obtain property (b).

\subsection{The strongly monotone case}

Let us recall that the operator $\nabla \phi+A$ is called strongly monotone over bounded sets if, for all positive real number $R$, there exists a continuous function $w_{R}: \mathbb{R}_{+} \rightarrow \mathbb{R}_{+}$verifying

$$
w_{R}\left(t_{n}\right) \rightarrow 0 \Rightarrow t_{n} \rightarrow 0
$$

and such that $(u, v) \in \mathcal{H}^{2}$ with $|u| \leq R$ and $|v| \leq R$ yields

$$
\langle(\nabla \phi+A) u-(\nabla \phi+A) v, u-v\rangle \geq w_{R}(|u-v|) .
$$

Clearly, this property implies that $S:=(\nabla \phi+A)^{-1}(0)$ contains at most one element and it holds if, for instance, $\nabla \phi$ or $A$ is strongly monotone over bounded sets (with the other operator being assumed to be monotone).

Proposition 2.1. Under the assumptions of Theorem 2.1, assuming moreover that $\nabla \phi+A$ is strongly monotone over bounded sets, then the solution $u(t)$ of (2.1) strongly converges as $t$ goes to $+\infty$ towards the unique element of $S:=(\nabla \phi+A)^{-1}(0)$.

Proof. By Theorem 2.1, we have $u, \dot{u} \in L^{\infty}([0,+\infty) ; \mathcal{H})$. Set $R:=\sup _{t \geq 0}|u(t)|+|p|$, with $p \in S$, and combine $(2.14)$ and $(2.32)$, to obtain $\ddot{h}(t)+w_{R}(|u(t)-p|) \leq|\dot{u}(t)|^{2}$, where $h(t):=(1 / 2)|u(t)-p|^{2}$. After integration, observing that $\dot{h} \in L^{\infty}([0,+\infty) ; \mathcal{H})$ and recalling that $\dot{u} \in L^{2}([0,+\infty) ; \mathcal{H})$ (Thm. 2.1, (i2)), we deduce that 
$\int_{0}^{+\infty} w_{R}(|u(s)-p|) \mathrm{d} s<+\infty$. On the other hand, by Theorem 2.1, (i4), $\lim _{t \rightarrow+\infty}|u(t)-p|$ exists, which by continuity of $w_{R}$ implies that $\lim _{t \rightarrow+\infty} w_{R}(|u(t)-p|)$ exists. Hence, $\lim _{t \rightarrow+\infty} w_{R}(|u(t)-p|)=0$. Then use the property of $w_{R}\left(w_{R}\left(t_{n}\right) \rightarrow 0 \Rightarrow t_{n} \rightarrow 0\right)$ to obtain $\lim _{t \rightarrow+\infty}|u(t)-p|=0$, which ends the proof.

\subsection{Some particular cases}

Let us specialize Theorem 2.1 and Proposition 2.1 to some important particular situations.

(a) Let us first take the non-potential operator $A$ equal to zero. Then notice that the null operator is $\lambda$-cocoercive for any $\lambda>0$. By taking $\lambda>\left(1 / \gamma^{2}\right)$, we can apply Theorem 2.1. Owing to the fact that no restrictive assumption is made on the potential part, we obtain the Alvarez theorem [2]:

Corollary 2.1. Let $\phi: \mathcal{H} \rightarrow \mathbb{R}$ be a convex differentiable function whose gradient $\nabla \phi$ is Lipschitz continuous on the bounded subsets of $\mathcal{H}$, and let $\gamma>0$. Let us assume that $S:=\operatorname{argmin}_{\mathcal{H}} \phi$ is nonempty. Then, for each initial data $u_{0}$ and $v_{0}$ in $\mathcal{H}$, the unique solution $u \in C^{2}([0,+\infty) ; \mathcal{H})$ of

$$
\ddot{u}(t)+\gamma \dot{u}(t)+\nabla \phi(u(t))=0,
$$

with initial conditions $u(0)=u_{0}$ and $\dot{u}(0)=v_{0}$, satisfies:

(i1) there exists $u_{\infty} \in S$ such that $u(t) \rightarrow u_{\infty}$ weakly in $\mathcal{H}$ as $t \rightarrow+\infty$;

(i2) $\dot{u} \in L^{2}(0,+\infty ; \mathcal{H}) ; \lim _{t \rightarrow+\infty}|\dot{u}(t)|=0$.

(b) Operators of the form $A=I-T$, where $T: \mathcal{H} \rightarrow \mathcal{H}$ is a contraction, play a central role in the realm of fixed point theory and constrained optimization. Let us verify that $A$ is $(1 / 2)$-cocoercive. Indeed, by setting

$$
E:=\langle(u-T u)-(v-T v), u-v\rangle-\frac{1}{2}|(u-T u)-(v-T v)|^{2},
$$

we obviously have

$$
\begin{aligned}
E & =\langle(u-v)-(T u-T v), u-v\rangle-\frac{1}{2}|(u-v)-(T u-T v)|^{2}, \\
& =|u-v|^{2}-\langle T u-T v, u-v\rangle-\frac{1}{2}|u-v|^{2}-\frac{1}{2}|T u-T v|^{2}+\langle(u-v), T u-T v\rangle, \\
& =\frac{1}{2}\left(|u-v|^{2}-|T u-T v|^{2}\right),
\end{aligned}
$$

which, by contraction property of $T$, is nonnegative. Thus we can take $\lambda=1 / 2$, and condition (1.5) boils down to $\gamma>\sqrt{2}$. Applying Theorem 2.1 to this situation, we obtain the following result (see [4], Thm. 3.2):

Corollary 2.2. Let $T: \mathcal{H} \rightarrow \mathcal{H}$ be a contraction and $\gamma>\sqrt{2}$. Let us assume that $S:=\operatorname{Fix} T=\{v \in \mathcal{H}: T v=v\}$ is nonempty. Then, for each initial data $u_{0}$ and $v_{0}$ in $\mathcal{H}$, the unique solution $u \in C^{2}([0,+\infty) ; \mathcal{H})$ of

$$
\ddot{u}(t)+\gamma \dot{u}(t)+u(t)-T(u(t))=0,
$$

with initial data $u(0)=u_{0}$ and $\dot{u}(0)=v_{0}$, satisfies:

(i1) there exists $u_{\infty} \in$ FixT such that $u(t) \rightarrow u_{\infty}$ weakly in $\mathcal{H}$ as $t \rightarrow+\infty$;

(i2) $\dot{u} \in L^{2}(0,+\infty ; \mathcal{H}) ; \lim _{t \rightarrow+\infty}|\dot{u}(t)|=\lim _{t \rightarrow+\infty}|\ddot{u}(t)|=0$.

(c) When working with a general maximal monotone operator $A: \mathcal{H} \rightarrow \mathcal{H}$, let us observe that, for every $\lambda>0$, its Yosida approximation $A_{\lambda}$ is $\lambda$-cocoercive. We recall that $A_{\lambda}=\frac{1}{\lambda}\left(I-J_{\lambda}^{A}\right)$, where $J_{\lambda}^{A}:=(I+\lambda A)^{-1}$ is the resolvent operator of index $\lambda$ of $A$. The operator $J_{\lambda}^{A}$ is everywhere defined, single-valued (it is a contraction) and $A_{\lambda} w \in\left(A \circ J_{\lambda}^{A}\right) w$ for any $w \in \mathcal{H}$ (see [20]). Let $(u, v) \in \mathcal{H}^{2}$ and set $E:=\left\langle A_{\lambda} u-A_{\lambda} v, u-v\right\rangle$. Noticing that $u=J_{\lambda}^{A} u+\lambda A_{\lambda} u$ and $v=J_{\lambda}^{A} v+\lambda A_{\lambda} v$, and recalling that $A_{\lambda} u \in A\left(J_{\lambda}^{A} u\right)$ and $A_{\lambda} v \in A\left(J_{\lambda}^{A} v\right)$, we immediately obtain

$$
E=\left\langle A_{\lambda} u-A_{\lambda} v,\left(J_{\lambda}^{A} u-J_{\lambda}^{A} v\right)+\lambda\left(A_{\lambda} u-A_{\lambda} v\right)\right\rangle \geq \lambda\left|A_{\lambda} u-A_{\lambda} v\right|^{2},
$$


which proves that $A_{\lambda}$ is $\lambda$-cocoercive. Observing that $A$ and $A_{\lambda}$ have the same zeroes, and as a straight consequence of Theorem 2.1, we reach the following result:

Corollary 2.3. Let $A: \mathcal{H} \rightarrow \mathcal{H}$ be a general maximal monotone operator and let $\gamma>0$ and $\lambda>0$ be such that $\lambda \gamma^{2}>1$. Let us assume that $S:=A^{-1}(0)$, the set of zeroes of $A$, is nonempty. Then, for each initial data $u_{0}$ and $v_{0}$ in $\mathcal{H}$, the unique solution $u \in C^{2}([0,+\infty) ; \mathcal{H})$ of

$$
\ddot{u}(t)+\gamma \dot{u}(t)+A_{\lambda}(u(t))=0,
$$

with initial data $u(0)=u_{0}$ and $\dot{u}(0)=v_{0}, A_{\lambda}$ being the Yosida approximation of index $\lambda$ of A, satisfies:

(i1) there exists $u_{\infty} \in S$ such that $u(t) \rightarrow u_{\infty}$ weakly in $\mathcal{H}$ as $t \rightarrow+\infty$;

(i2) $\dot{u} \in L^{2}(0,+\infty ; \mathcal{H}) ; \lim _{t \rightarrow+\infty}|\dot{u}(t)|=0$.

Let us examine an interesting consequence of Corollary 2.3 regarding numerical schemes. From resolvent equation (see [20], Prop. 2.6), we have $\left(A_{\lambda}\right)_{\mu}=A_{\lambda+\mu}$ whenever $\lambda>0$ and $\mu>0$. Then it can be easily derived that, for any $v \in \mathcal{H}$,

$$
J_{\mu}^{A_{\lambda}} v=\frac{\lambda}{\lambda+\mu} v+\frac{\mu}{\lambda+\mu} J_{\lambda+\mu}^{A} v
$$

Implicit time discretization of (2.35) naturally leads to a second order relaxed proximal algorithm, whose trajectories (sequences) converge to the set of zeroes $A^{-1}(0)$, for $A$ a general maximal monotone operator, see [3] and references herein.

\subsection{Condition (1.5) is sharp}

Example 2.1. On $\mathbb{C}$ endowed with the standard real Hilbert structure associated to the inner product $\langle u, v\rangle=$ $\operatorname{Re}(u \bar{v})$, we consider the following equation

$$
\ddot{u}(t)+\gamma \dot{u}(t)+A u(t)=0
$$

where $A u:=\left(w^{2}-i \gamma w\right) u$ with $\gamma>0$ and $w>0$. It is trivially checked that $A$ is $\lambda$-cocoercive with $\lambda=$ $1 /\left(w^{2}+\gamma^{2}\right)$. A solution of (2.37) is given by the standard harmonic oscillator $u(t)=\mathrm{e}^{\mathrm{i} w t}$. It appears that $u$ is bounded but not convergent for any $w>0$. Moreover, by letting $w \rightarrow 0^{+}$we get $\lambda \gamma^{2} \rightarrow 1^{-}$, which shows that the condition $\lambda \gamma^{2}<1$ is not sufficient for the convergence of (2.1) in general.

Example 2.2. Let $B: \mathbb{R}^{2} \rightarrow \mathbb{R}^{2}$ be the $\pi / 2$ rotation of center $(0,0)$ and denote by $B_{\lambda}$ its Yosida approximation of parameter $\lambda>0$. It is a classical matter to see that $B$ and $B_{\lambda}$ are linear operators whose matrixes in the canonical basis of $\mathbb{R}^{2}$ are defined by $B=\left(\begin{array}{cc}0 & -1 \\ 1 & 0\end{array}\right)$ and $B_{\lambda}=\frac{1}{1+\lambda^{2}}\left(\begin{array}{cc}\lambda & -1 \\ 1 & \lambda\end{array}\right)$. Given $\gamma>0$, we then consider the dynamical system

$$
\ddot{X}(t)+\gamma \dot{X}(t)+B_{\lambda} X(t)=0 .
$$

The operator $B_{\lambda}$ is $\lambda$-cocoercive, as follows from general properties of Yosida approximation, or by a direct elementary computation. It can be checked that (2.38) will have non-convergent solutions if

$$
\sqrt{\gamma^{4}-\frac{8 \gamma^{2} \lambda}{1+\lambda^{2}}+\frac{16}{1+\lambda^{2}}} \geq\left(\gamma^{2}+\frac{4 \lambda}{1+\lambda^{2}}\right)
$$

Theses solutions are bounded if equality holds in (2.39) and unbounded otherwise. In addition, setting $\lambda=\theta / \gamma^{2}$ (with $\theta \geq 0$ ), and after an easy computation, we obtain that $(2.39)$ is equivalent to $\gamma^{4}(1-\theta) \geq \theta^{3}$. Hence $(2.38)$ has non-convergent trajectories when $\theta \in[0,1)$, which means that $\lambda \gamma^{2}<1$ does not ensure convergence of dynamical system $(2.1)$. 


\subsection{Invariance of condition (1.5) with respect to time rescaling}

Given some positive real parameter $k$, let us consider the time rescaling $t=k s$. For any trajectory $u($. of $(2.1)$, the rescaled trajectory $v(s)=u(k s)$ satisfies

$$
\ddot{v}(s)+\gamma k \dot{v}(s)+k^{2} \nabla \phi(v(s))+k^{2} A(v(s))=0 .
$$

Let us suppose that $A$ is a $\lambda$-cocoercive maximal monotone operator. One can easily verify that $k^{2} A$ is a $\left(\lambda / k^{2}\right)$ cocoercive maximal monotone operator. Clearly, this time rescaling does not change the asymptotic convergence properties of the trajectories. Indeed, the condition insuring the convergence of the trajectories, namely $\lambda \gamma^{2}>1$, remains invariant under this time rescaling, as shown by the following relation: $\left(\lambda / k^{2}\right) \times(\gamma k)^{2}=\lambda \gamma^{2}$. This elementary observation tells us that the condition $\lambda \gamma^{2}>1$ makes sense from a physical point of view.

\section{Asymptotic stabilization by Tikhonov Regularization methods}

In order to correct some drawbacks of system (1.3), namely the weak (and possibly not strong) asymptotic convergence property of its trajectories, and the dependence of the limit equilibrium on the initial data, we introduce in the equation a Tikhonov-like regularization term with a vanishing coefficient. To be more precise, we are going to establish strong asymptotic convergence results regarding the solution of the non-autonomous system

$$
\left[\begin{array}{l}
\ddot{u}(t)+\gamma \dot{u}(t)+\nabla \phi(u(t))+A(u(t))+\epsilon(t) \nabla \Theta(u(t))=0, \\
u(0)=u_{0}, \quad \dot{u}(0)=v_{0}
\end{array}\right.
$$

where $\gamma>0$ and $\left(u_{0}, v_{0}\right)$ in $\mathcal{H}^{2}$ are arbitrary given initial data. We make the following assumptions:

(H1) $\phi: \mathcal{H} \rightarrow \mathbb{R}$ is a convex differentiable function whose gradient $\nabla \phi$ is Lipschitz continuous on bounded sets;

(H2) $A: \mathcal{H} \rightarrow \mathcal{H}$ is maximal monotone and $\lambda$-cocoercive for some $\lambda>0$;

(H3) $\epsilon:[0,+\infty) \rightarrow(0,+\infty)$ is a positive mapping of class $C^{1}$ such that $\epsilon(t) \rightarrow 0$ as $t \rightarrow+\infty$. Moreover, $\dot{\epsilon}$ is supposed to be bounded and Lipschitz continuous;

(H4) $\Theta: \mathcal{H} \rightarrow \mathbb{R}$ is convex, differentiable, and bounded from below on $\mathcal{H}$. Moreover, its derivative $\nabla \Theta$ is supposed to be $\delta$-Lipschitz continuous (LC) and $\eta$-strongly monotone (SM), with $\delta>0$ and $\eta>0$, i.e.,

$$
\begin{aligned}
& \text { (LC) }|\nabla \Theta(u)-\nabla \Theta(v)| \leq \delta|u-v| \quad \forall u, v \in \mathcal{H}, \\
& \text { (SM) }\langle\nabla \Theta(u)-\nabla \Theta(v), u-v\rangle \geq \eta|u-v|^{2} \quad \forall u, v \in \mathcal{H} .
\end{aligned}
$$

At once, we claim the main result of this section.

Theorem 3.1. Let us suppose that (H1)-(H4) hold with $S:=(A+\nabla \phi)^{-1}(0) \neq \emptyset$ and with $\lambda \gamma^{2}>1$. Let us assume moreover that $\epsilon(t)$ is decreasing and converges slowly to zero as $t \rightarrow+\infty$ in the following sense:

$$
\int_{0}^{+\infty} \epsilon(s) \mathrm{d} s=+\infty .
$$

Then, for any $u_{0}$ and $v_{0}$ in $\mathcal{H}$, there exists a unique solution $u \in C^{2}([0,+\infty) ; \mathcal{H})$ of $(3.1)$ which satisfies $u(t) \rightarrow u_{*}$ strongly in $\mathcal{H}$ as $t \rightarrow+\infty$, where $u_{*}$ is the unique solution of the variational inequality: find $u_{*} \in S$ such that

$$
\left\langle\nabla \Theta\left(u_{*}\right), v-u_{*}\right\rangle \geq 0 \quad \forall v \in S .
$$

Before proving Theorem 3.1, we introduce a series of preliminary results. For the sake of simplicity, as in Section 2, we first reformulate (3.1) as follows. 
Remark 3.1. Consider the operator $B$ and the function $\Phi$ given in Remark 2.3, namely $\Phi=\phi+\langle d,$.$\rangle and$ $B=A-d$, for some element $d \in \mathcal{H}$. We recall that $S$ can be rewritten as $S=\{p \in \mathcal{H}, \quad \nabla \Phi(p)=0, \quad B p=0\}$. It is also obvious that the solution $u$ of (3.1) satisfies $\ddot{u}+\gamma \dot{u}+\nabla \Phi(u)+B u+\epsilon(t) \nabla \Theta(u)=0$. Given $p \in S$ and setting $v=u-p$, we can see that the above equation can be rewritten as

$$
\ddot{v}+\gamma \dot{v}+\nabla \bar{\phi}(v)+\bar{A} v+\epsilon \nabla \bar{\Theta}(v)=0
$$

where $\bar{A} v=B(v+p), \bar{\phi}(v)=\Phi(v+p)-\Phi(p)$ and $\bar{\Theta}(v)=\Theta(v+p)$. Regarding the properties of $\bar{A}$ and $\bar{\phi}$ under conditions (H1)-(H2), we also recall (see Rem. 2.4) that $\bar{A}$ is $\lambda$-cocoercive, $\bar{\phi}$ is convex, its gradient is Lipschitz continuous on the bounded subsets of $\mathcal{H}$, and it holds that $\nabla \bar{\phi}(0)=0$ and $\bar{A} 0=0$.

To begin with, we establish a key estimate on the trajectories of (3.3).

Lemma 3.1. Under conditions (H1)-(H4), and for any $p \in S$, the solution $v$ of (3.3) satisfies

$$
\lambda G(t)+w(t)+\left(\lambda \gamma^{2}-1\right)|\dot{v}(t)|^{2}+\dot{\Gamma}_{1}(t) \leq-\epsilon(t)\langle\nabla \bar{\Theta}(v(t)), v(t)\rangle+2 \lambda \gamma \dot{\epsilon}(t) \bar{\Theta}_{c}(v(t))
$$

where $w($.$) and G($.$) are defined by$

$$
w(t)=\langle\nabla \bar{\phi}(v(t)), v(t)\rangle, G(t):=|\bar{A}(v(t))+\gamma \dot{v}(t)|^{2},
$$

$\dot{\Gamma}_{1}(\cdot)$ denotes the first derivative of the mapping $\Gamma_{1}(\cdot)$ given by

with

$$
\Gamma_{1}(t)=\dot{h}(t)+\gamma h(t)+\lambda \gamma\left(|\dot{v}(t)|^{2}+2 \bar{\phi}(v(t))+2 \epsilon(t) \bar{\Theta}_{c}(v(t))\right)
$$

$$
h(t)=(1 / 2)|v(t)|^{2}, \bar{\Theta}_{c}(v):=\bar{\Theta}(v)-\inf _{\mathcal{H}} \bar{\Theta} .
$$

Proof. Without ambiguity, in order to get simplified notations, we omit to write the variable $t$. Hence $v$ stands for $v(t)$, and so on. Using (3.3), we have

$$
\langle\nabla \bar{\phi}(v), v\rangle+\langle\bar{A} v, v\rangle=-\langle\ddot{v}+\gamma \dot{v}+\epsilon(t) \nabla \bar{\Theta}(v), v\rangle,
$$

which by $\lambda$-cocoerciveness of $\bar{A}$ together with $\bar{A} 0=0$ (see Rem. 3.1) and by the above definition of $w(t)$ amounts to

$$
\lambda|\bar{A} v|^{2}+w(t) \leq-\langle\ddot{v}+\gamma \dot{v}, v\rangle-\epsilon(t)\langle\nabla \bar{\Theta}(v), v\rangle
$$

Furthermore, from $h=(1 / 2)|v|^{2}$, we have $\dot{h}=\langle\dot{v}, v\rangle$ and $\ddot{h}=\langle\ddot{v}, v\rangle+|\dot{v}|^{2}$, so that $\ddot{h}+\gamma \dot{h}=\langle\ddot{v}+\gamma \dot{v}, v\rangle+|\dot{v}|^{2}$. This combined with (3.6) gives

$$
\lambda|\bar{A} v|^{2}+w(t)+(\ddot{h}+\gamma \dot{h}) \leq|\dot{v}|^{2}-\epsilon(t)\langle\nabla \bar{\Theta}(v), v\rangle
$$

Let us reexpress $|\bar{A} v|^{2}$ with the help of (3.3):

$$
\begin{aligned}
|\bar{A} v|^{2} & =|\ddot{v}+\nabla \bar{\phi}(v)+\epsilon(t) \nabla \bar{\Theta}(v)+\gamma \dot{v}|^{2} \\
& =G(t)+\gamma^{2}|\dot{v}|^{2}+2 \gamma\langle\dot{v}, \ddot{v}+\nabla \bar{\phi}(v)+\epsilon(t) \nabla \bar{\Theta}(v)\rangle
\end{aligned}
$$

where $G(t):=|\ddot{v}+\nabla \bar{\phi}(v)+\epsilon(t) \nabla \bar{\Theta}(v)|^{2}$ (hence $G(t)=|\bar{A} v(t)+\gamma \dot{v}(t)|^{2}$ ).

Set $\bar{\Theta}_{c}()=.\bar{\Theta}()-.\inf _{\mathcal{H}} \bar{\Theta}, Q(t)=(1 / 2)|\dot{v}|^{2}+\bar{\phi}(v)$, and rewrite $(3.8)$ as

$$
|\bar{A} v|^{2}=G(t)+\gamma^{2}|\dot{v}|^{2}+2 \gamma \frac{\mathrm{d}}{\mathrm{d} t}\left(Q(t)+\epsilon(t) \bar{\Theta}_{c}(v)\right)-2 \gamma \dot{\epsilon}(t) \bar{\Theta}_{c}(v) .
$$

Replacing this last expression in (3.7) we obtain

$$
w(t)+(\ddot{h}+\gamma \dot{h})+\lambda\left(G(t)+\gamma^{2}|\dot{v}|^{2}+2 \gamma \frac{\mathrm{d}}{\mathrm{d} t}\left(Q(t)+\epsilon(t) \bar{\Theta}_{c}(v)\right)-2 \gamma \dot{\epsilon}(t) \bar{\Theta}_{c}(v)\right) \leq|\dot{v}|^{2}-\epsilon(t)\langle\nabla \bar{\Theta}(v), v\rangle
$$


or equivalently

$$
w(t)+\lambda G(t)+\left(\lambda \gamma^{2}-1\right)|\dot{v}|^{2}+\dot{\Gamma}_{1}(t) \leq-\epsilon(t)\langle\nabla \bar{\Theta}(v), v\rangle+2 \lambda \gamma \dot{\epsilon}(t) \bar{\Theta}_{c}(v),
$$

where the function $\Gamma_{1}(\cdot)$ is defined by

$$
\begin{aligned}
\Gamma_{1}(t) & =\dot{h}(t)+\gamma h(t)+2 \lambda \gamma\left(Q(t)+\epsilon(t) \bar{\Theta}_{c}(v(t))\right) \\
& =\dot{h}(t)+\gamma h(t)+\lambda \gamma\left(|\dot{v}(t)|^{2}+2 \bar{\phi}(v(t))+2 \epsilon(t) \bar{\Theta}_{c}(v(t))\right)
\end{aligned}
$$

which leads to (3.4).

We also need the following variant of Gronwall's inequality, see [24] (Lem. 1).

Lemma 3.2. Let $\psi:[0,+\infty) \rightarrow \mathbb{R}$ be absolutely continuous with

$$
\dot{\psi}(t)+\epsilon(t) \psi(t) \leq \epsilon(t) g(t), \quad \text { a.e. },
$$

where $g(t)$ is bounded and $\epsilon(t) \geq 0$ with $\epsilon \in L_{\mathrm{loc}}^{1}\left(\mathbb{R}_{+}\right)$. Then the function $\psi(t)$ is bounded from above and if $\int_{0}^{+\infty} \epsilon(\tau) \mathrm{d} \tau=+\infty$ we have $\lim \sup _{t \rightarrow+\infty} \psi(t) \leq \lim \sup _{t \rightarrow+\infty} g(t)$.

Proof. Let $\kappa_{s}:=\sup \{g(t): t \geq s\}$ so that $\dot{\psi}(t)+\epsilon(t)\left[\psi(t)-\kappa_{s}\right] \leq 0$ for $t \geq s$. Multiplying by $\exp \left(\int_{0}^{t} \epsilon(\tau) \mathrm{d} \tau\right)$ and integrating over $[s, t]$ we get $\left[\psi(t)-\kappa_{s}\right] \leq\left[\psi(s)-\kappa_{s}\right] \exp \left(-\int_{s}^{t} \epsilon(\tau) \mathrm{d} \tau\right)$. It follows that $\psi(t)$ is bounded from above and, if $\int_{0}^{+\infty} \epsilon(\tau) \mathrm{d} \tau=+\infty$, by letting $t \rightarrow+\infty$ in the above estimate we obtain $\lim \sup _{t \rightarrow+\infty} \psi(t) \leq \kappa_{s}$. Letting $s \rightarrow+\infty$ in this last inequality yields $\lim \sup _{t \rightarrow+\infty} \psi(t) \leq \lim \sup _{t \rightarrow+\infty} g(t)$.

At once we prove the boundedness of the trajectory given by (3.1).

Lemma 3.3. Let conditions $(\mathrm{H} 1)-(\mathrm{H} 4)$ be satisfied with parameters such that

$$
\lambda \gamma^{2}>1 \text {, and } \dot{\epsilon}(t) \leq 0 \text { for all } t \geq 0 \text {. }
$$

Then for any solution $u$ of (3.1) it holds that

$$
u \in L^{\infty}([0,+\infty), \mathcal{H}) \text { and } \dot{u} \in L^{\infty}([0,+\infty), \mathcal{H}) .
$$

If in addition $\int_{0}^{+\infty} \epsilon(s) \mathrm{d} s=+\infty$, then the following properties are equivalent:

(i1) any weak cluster point of $u(t)$ for $t \rightarrow+\infty$ belongs to $S:=(\nabla \phi+A)^{-1}(0)$;

(i2) $\liminf \operatorname{int}_{t \rightarrow+\infty}\left\langle\nabla \Theta\left(u_{*}\right), u(t)-u_{*}\right\rangle \geq 0$;

(i3) $u(t) \rightarrow u_{*}$ strongly;

where $u_{*}$ is the unique solution of (1.10).

Proof. Let us consider $v=u-u_{*}$ in Lemma 3.1, use the same notations and set $z():.=\langle\nabla \bar{\Theta}(v), v\rangle$. Recall that $\Gamma_{1}=\dot{h}+\gamma h+\lambda \gamma\left(|\dot{v}|^{2}+2 \bar{\phi}(v)+2 \epsilon(t) \bar{\Theta}_{c}(v)\right)$. With these notations, by using $\dot{\epsilon} \leq 0$ and $\bar{\Theta}_{c}(v) \geq 0$, Lemma 3.1 gives

$$
\lambda G+w+\left(\lambda \gamma^{2}-1\right)|\dot{v}|^{2}+\dot{\Gamma}_{1} \leq-\epsilon z .
$$

For any real values $\nu>0$ and $\rho \in(0,1)$, by definition of $\Gamma_{1}$ we also have

$$
\begin{aligned}
\nu \Gamma_{1} & =\nu \dot{h}+(\nu \gamma) h+(\nu \lambda \gamma)|\dot{v}|^{2}+2(\nu \lambda \gamma) \bar{\phi}(v)+2(\nu \lambda \gamma) \epsilon(t) \bar{\Theta}_{c}(v) \\
& =\left(\rho \eta h+\nu 2 \lambda \gamma \epsilon \bar{\Theta}_{c}(v)\right)+(\nu \gamma-\rho \eta) h+\nu \lambda \gamma|\dot{v}|^{2}+\nu \dot{h}+2(\lambda \gamma \nu) \bar{\phi}(v)
\end{aligned}
$$

Hence, adding $-z$ to each side of the above equality, we equivalently obtain

$$
-z=-\nu \Gamma_{1}+\left(-z+\rho \eta h+\nu 2 \lambda \gamma \epsilon \bar{\Theta}_{c}(v)\right)+(\nu \gamma-\rho \eta) h+\nu \lambda \gamma|\dot{v}|^{2}+\nu \dot{h}+2(\lambda \gamma \nu) \bar{\phi}(v),
$$


which by (3.9) amounts to

$$
\begin{aligned}
\lambda G+w+\left(\lambda \gamma^{2}-1\right)|\dot{v}|^{2}+\dot{\Gamma}_{1}+\nu \epsilon \Gamma_{1} \leq \epsilon\left(-z+\rho \eta h+\nu 2 \lambda \gamma \epsilon \bar{\Theta}_{c}(v)\right)+\epsilon & (\nu \gamma-\rho \eta) h \\
& +\nu \epsilon \lambda \gamma|\dot{v}|^{2}+\nu \epsilon \dot{h}+2 \epsilon(\lambda \gamma \nu) \bar{\phi}(v) .
\end{aligned}
$$

By convexity of $\bar{\phi}$, recalling that $w=\langle\nabla \bar{\phi}(v), v\rangle$ and $\bar{\phi}(0)=0$, we have $\bar{\phi}(v) \leq w(t)$, while by Young's inequality $\dot{h}=\langle\dot{v}, v\rangle \leq(1 / 2)|\dot{v}|^{2}+h$. As a result, combining these last two inequalities with (3.10), we deduce that

$$
\begin{aligned}
\lambda G+(1-2 \lambda \gamma \nu \epsilon) w+\left[\lambda \gamma^{2}-1-\nu \epsilon(\lambda \gamma+1 / 2)\right]|\dot{v}|^{2}+ & \dot{\Gamma}_{1}+\nu \epsilon \Gamma_{1} \\
& \leq \epsilon\left(-z+\rho \eta h+2 \lambda \gamma \nu \epsilon \bar{\Theta}_{c}(v)\right)+\epsilon(\nu \gamma+\nu-\rho \eta) h .
\end{aligned}
$$

Hence, for $\nu=(\rho \eta) /(\gamma+1)$, we equivalently obtain

$$
\lambda G+(1-2 \lambda \gamma \nu \epsilon) w+\left(\lambda \gamma^{2}-1-\nu \epsilon(\lambda \gamma+1 / 2)\right)|\dot{v}|^{2}+\dot{\Gamma}_{1}+\nu \epsilon \Gamma_{1} \leq \epsilon\left(-z+\rho \eta h+2 \nu \lambda \gamma \epsilon \bar{\Theta}_{c}(v)\right)
$$

Moreover, it is assumed that $\lambda \gamma^{2}-1>0$ and $\epsilon(t) \rightarrow 0$ as $t \rightarrow+\infty$. Therefore, for $t$ large enough, say $t \geq t_{0}$, we have $\lambda \gamma^{2}-1-\nu \epsilon(t)(\lambda \gamma+1 / 2) \geq 0$ and $1-2 \lambda \gamma \nu \epsilon(t) \geq 0$. By (3.12) and positivity of $G(\cdot)$ (and omitting the variable $t$ ), we deduce that for $t$ large enough

$$
\dot{\Gamma}_{1}+\nu \epsilon \Gamma_{1} \leq \epsilon\left(-z+\rho \eta h+2 \lambda \gamma \nu \epsilon \bar{\Theta}_{c}(v)\right)
$$

By $z():.=\langle\nabla \bar{\Theta}(v), v\rangle$ and convexity of $\bar{\Theta}$

while by $\eta$-strong-monotonicity of $\nabla \bar{\Theta}$

$$
z \geq \bar{\Theta}_{c}(v)-\bar{\Theta}_{c}(0)
$$

$$
z \geq\langle\nabla \bar{\Theta}(0), v\rangle+2 \eta h .
$$

Let us apply Young's inequality to this latter inequality

$$
z \geq-(1 / 2 \eta)|\nabla \bar{\Theta}(0)|^{2}+\eta h .
$$

Recalling that $\rho \in(0,1)$, and using (3.14) and (3.16), we obtain

$$
z \geq(1-\rho)\left(\bar{\Theta}_{c}(v)-\bar{\Theta}_{c}(0)\right)-\rho(1 / 2 \eta)|\nabla \bar{\Theta}(0)|^{2}+\rho \eta h,
$$

which by (3.13) amounts to

$$
\dot{\Gamma}_{1}+\nu \epsilon \Gamma_{1} \leq \epsilon\left((1-\rho) \bar{\Theta}_{c}(0)+\rho(1 / 2 \eta)|\nabla \bar{\Theta}(0)|^{2}\right)-\epsilon((1-\rho)-2 \lambda \gamma \nu \epsilon) \bar{\Theta}_{c}(v) .
$$

Observing that $(1-\rho)-2 \lambda \gamma \nu \epsilon(t) \geq 0$ for $t \geq t_{1}$ large enough, we obtain

$$
\dot{\Gamma}_{1}+\nu \epsilon \Gamma_{1} \leq \epsilon\left((1-\rho) \bar{\Theta}_{c}(0)+\rho(1 / 2 \eta)|\nabla \bar{\Theta}(0)|^{2}\right)
$$

Applying Lemma 3.2 we deduce that $\Gamma_{1}$ is bounded. By definition of $\Gamma_{1}$ and convexity of $\bar{\phi}$ (note that $0 \leq \bar{\phi}(v)$, as $\bar{\phi}(0)=0$ and $\nabla \bar{\phi}(0)=0)$ we have

$$
\dot{h}(t)+\gamma h(t)+\lambda \gamma|\dot{v}(t)|^{2} \leq \Gamma_{1}(t)
$$

Hence there exists a positive constant $C$ such that $t$ large enough, say $t \geq t_{2}$, yields

$$
\dot{h}(t)+\gamma h(t)+\lambda \gamma|\dot{v}(t)|^{2} \leq C,
$$


which obviously implies that $h$ is bounded on $[0,+\infty[$, and so is $u$ :

$$
\sup _{t \in[0,+\infty)}|u(t)|<+\infty \text {. }
$$

From $\dot{h}=\langle\dot{v}, v\rangle,(3.21)$ and $(3.22)$ we also derive the boundedness of $\dot{u}($ as $\dot{u}=\dot{v}$ ):

$$
\sup _{t \in[0,+\infty)}|\dot{u}(t)|<+\infty .
$$

Let us now prove the equivalences. Note that (i1) $\Rightarrow$ (i2) follows from (1.10), while (i3) $\Rightarrow$ (i1) is obvious. It remains to prove (i2) $\Rightarrow$ (i3). Let us assume that (i2) holds. Again from (3.13) and (3.15), and by $\rho \in(0,1)$, we immediately have

$$
\dot{\Gamma}_{1}+\nu \epsilon \Gamma_{1} \leq \epsilon\left(-\langle\nabla \bar{\Theta}(0), v\rangle+2 \lambda \gamma \nu \epsilon \bar{\Theta}_{c}(v)\right) .
$$

Consequently, applying Lemma 3.2 amounts to

$$
\begin{aligned}
\lim \sup _{t \rightarrow+\infty} \Gamma_{1}(t) & \leq(1 / \nu) \limsup _{t \rightarrow+\infty}\left[-\langle\nabla \bar{\Theta}(0), v(t)\rangle+2 \lambda \gamma \nu \epsilon(t) \bar{\Theta}_{c}(v(t))\right] \\
& =-(1 / \nu) \liminf _{t \rightarrow+\infty}\langle\nabla \bar{\Theta}(0), v(t)\rangle .
\end{aligned}
$$

We also recall that $\langle\nabla \bar{\Theta}(0), v\rangle=\left\langle\nabla \Theta\left(u_{*}\right), u-u_{*}\right\rangle$. It is then obvious that (i2) leads to $\lim \sup _{t \rightarrow+\infty} \Gamma_{1}(t) \leq 0$, which by (3.20) entails $\lim \sup _{t \rightarrow+\infty}(\dot{h}(t)+\gamma h(t)) \leq 0$. Then, it is easily checked (one can apply Lem. 3.2) that this inequality implies $\lim \sup _{t \rightarrow+\infty} h(t)=0$, namely (i3).

We are now in position to prove convergence in norm of the solution trajectory $u$ of (3.1) towards a special zero of $\nabla \phi+A$.

Proof of Theorem 3.1. From Lemma 3.3 we know that

$$
u \in L^{\infty}([0,+\infty) ; \mathcal{H}) \text { and } \dot{u} \in L^{\infty}([0,+\infty) ; \mathcal{H}) .
$$

Let us choose $p=u_{*}$ in Lemma 3.1 (hence $v=u-u_{*}$ ). Keeping the same notations, we have

$$
\lambda G(t)+w(t)+\left(\lambda \gamma^{2}-1\right)|\dot{v}(t)|^{2}+\dot{\Gamma}_{1}(t) \leq-\epsilon(t)\langle\nabla \bar{\Theta}(v(t)), v(t)\rangle,
$$

where

$$
\begin{gathered}
\Gamma_{1}(t)=\dot{h}(t)+\gamma h(t)+\lambda \gamma\left(|\dot{v}(t)|^{2}+2 \bar{\phi}(v(t))+2 \epsilon(t) \bar{\Theta}_{c}(v(t))\right), \\
w(t)=\langle\nabla \bar{\phi}(v(t)), v(t)\rangle \text { and } G(t):=|\bar{A}(v(t))+\gamma \dot{v}(t)|^{2} .
\end{gathered}
$$

The end of the proof is divided into two cases:

Case 1. Assume that there exists some time $t_{0} \geq 0$ such that $\Gamma_{1}(\cdot)$ is nonincreasing on $\left[t_{0},+\infty\right)$. Recalling that $\Gamma_{1}$ is lower bounded (thanks to its definition and (3.25)), we deduce that $\Gamma_{1}(t)$ converges as $t \rightarrow+\infty$ to some real value $\alpha$. Let us show that $\lim _{t \rightarrow+\infty} \dot{\Gamma}_{1}(t)=0$. Indeed, for any real numbers $t$ and $s$ verifying $t \geq s \geq t_{0}$, we have

$$
\int_{s}^{t}\left|\dot{\Gamma}_{1}(\tau)\right| \mathrm{d} \tau=-\int_{s}^{t} \dot{\Gamma}_{1}(\tau) \mathrm{d} \tau=\Gamma_{1}(s)-\Gamma_{1}(t)
$$


Passing to the limit as $t \rightarrow+\infty$, we obtain

$$
\int_{s}^{+\infty}\left|\dot{\Gamma}_{1}(\tau)\right| \mathrm{d} \tau=\Gamma_{1}(s)-\alpha<+\infty .
$$

On the other hand, by using equation (3.1), together with (3.25), and assumption (H3) ( $\dot{\epsilon}$ is supposed to be bounded and Lipschitz continuous), we can observe that $\left|\dot{\Gamma}_{1}(\cdot)\right|$ is Lipschitz continuous. As a consequence $\dot{\Gamma}_{1}(t) \rightarrow 0$ as $t \rightarrow+\infty$.

Therefore, by (3.26), the boundedness of $u(\cdot)$, together with $\lambda \gamma^{2}-1>0$ and $\epsilon(t) \rightarrow 0$ as $t \rightarrow+\infty$, we deduce that $\lim _{t \rightarrow+\infty} w(t)=\lim _{t \rightarrow+\infty} G(t)=\lim _{t \rightarrow+\infty}|\dot{v}(t)|=0$. It follows that $\lim _{t \rightarrow+\infty} w(t)=0$ and $\lim _{t \rightarrow+\infty}|\bar{A} v(t)|=0($ as $G(t) \rightarrow 0$ and $|\dot{v}(t)| \rightarrow 0$ ). Hence, in light of Remark 2.5, we observe that any weak cluster point of $u$ belongs to $S$, which by Lemma 3.3 shows that $u(t) \rightarrow u_{*}$ strongly.

Case 2. Assume that there exists an increasing sequence $\left(l_{k}\right) \subset[0,+\infty)$ such that $\lim _{k \rightarrow+\infty} l_{k}=+\infty$ and $\dot{\Gamma}_{1}\left(l_{k}\right)>0$ for all $k \geq 0$. This allows us to introduce the mapping $s:\left[l_{0},+\infty\right) \rightarrow[0,+\infty)$ defined for $t$ large enough by

$$
s(t)=\sup \left\{\beta \leq t \quad \mid \quad \dot{\Gamma}_{1}(\beta)>0\right\}
$$

Let us establish that $\lim \sup _{t \rightarrow+\infty} \Gamma_{1}(t) \leq 0$. Let $\left(t_{n}\right) \subset[0,+\infty)$ be a nondecreasing sequence such that $\lim _{n \rightarrow+\infty} t_{n}=+\infty$, and set $s_{n}=s\left(t_{n}\right)$. It is obviously seen that $\left(s_{n}\right)$ is a nondecreasing sequence such that $s_{n} \rightarrow+\infty$ as $n \rightarrow+\infty,\left(\dot{\Gamma}_{1}\left(s_{n}\right)\right) \subset[0,+\infty)$, while by (3.26) we have

$$
\lambda G\left(s_{n}\right)+w\left(s_{n}\right)+\left(\lambda \gamma^{2}-1\right)\left|\dot{v}\left(s_{n}\right)\right|^{2} \leq-\epsilon\left(s_{n}\right)\left\langle\nabla \bar{\Theta}\left(v\left(s_{n}\right)\right), v\left(s_{n}\right)\right\rangle
$$

From this last inequality, keeping in mind that $\lambda \gamma^{2}-1>0$ and $\epsilon\left(s_{n}\right) \rightarrow 0$ as $n \rightarrow \infty$, we obviously deduce that

$$
\lim _{n \rightarrow+\infty}\left|\dot{u}\left(s_{n}\right)\right|=\lim _{n \rightarrow+\infty} w\left(s_{n}\right)=\lim _{n \rightarrow+\infty}\left|\bar{A} v\left(s_{n}\right)\right|=0 .
$$

By using $\epsilon\left(s_{n}\right) \in(0,+\infty)$, we additionally obtain

$$
\left\langle\nabla \bar{\Theta}\left(v\left(s_{n}\right)\right), v\left(s_{n}\right)\right\rangle \leq 0 .
$$

Clearly, (3.28) entails that any weak-cluster point of $u\left(s_{n}\right)$ belongs to $S$ (according to Rem. 2.5), while by (3.29) and using the $\eta$-strong monotonicity of $\nabla \Theta$, we obtain

$$
\eta\left|v\left(s_{n}\right)\right|^{2} \leq\left\langle\nabla \bar{\Theta}\left(v\left(s_{n}\right)\right)-\nabla \bar{\Theta}(0), v\left(s_{n}\right)\right\rangle \leq-\left\langle\nabla \bar{\Theta}(0), v\left(s_{n}\right)\right\rangle .
$$

Then, observing that $\langle\nabla \bar{\Theta}(0), v\rangle=\left\langle\nabla \Theta\left(u_{*}\right), u-u_{*}\right\rangle$, we get

$$
\limsup _{n \rightarrow+\infty}\left(\eta\left|v\left(s_{n}\right)\right|^{2}\right) \leq-\liminf _{n \rightarrow+\infty}\left\langle\nabla \bar{\Theta}(0), v\left(s_{n}\right)\right\rangle \leq 0
$$

this last inequality being obtained by passing to the limit on a weak convergent subsequence of $u\left(s_{n}\right)$, using the fact that any weak-cluster point of $u\left(s_{n}\right)$ belongs to $S$ and the definition (1.10) of $u_{*}$. Hence $u\left(s_{n}\right) \rightarrow u_{*}$ strongly, which together with (3.28) and the definition of $\Gamma_{1}$ implies that

$$
\lim _{n \rightarrow+\infty} \Gamma_{1}\left(s_{n}\right)=0
$$

In addition, using the definition of $s\left(t_{n}\right)$ we clearly have $s_{n} \leq t_{n}$ and $\dot{\Gamma}_{1}(t) \leq 0$ for $\left.t \in\right] s\left(t_{n}\right)$, $t_{n}[$ when $s\left(t_{n}\right) \neq t_{n}$. Let us write $\Gamma_{1}\left(t_{n}\right)=\Gamma_{1}\left(s_{n}\right)+\int_{s_{n}}^{t_{n}} \dot{\Gamma}_{1}(t) \mathrm{d} t \leq \Gamma_{1}\left(s_{n}\right)$, which by (3.30) yields $\lim \sup _{n \rightarrow \infty} \Gamma_{1}\left(t_{n}\right) \leq 0$. By convexity of $\bar{\phi}$, we also get $\dot{h}(t)+\gamma h(t) \leq \Gamma_{1}(t)$, which yields $\limsup _{n \rightarrow \infty}\left(\dot{h}_{(}\left(t_{n}\right)+\gamma h\left(t_{n}\right)\right) \leq 0$. 
This latter inequality being true for any nondecreasing sequence $\left(t_{n}\right)$ that converges to infinity, we conclude that $\lim \sup _{t \rightarrow+\infty}(\dot{h}(t)+\gamma h(t)) \leq 0$, which immediately leads to $u(t) \rightarrow u_{*}$ strongly.

The case $\Theta(v)=\frac{1}{2}|v|^{2}$, which corresponds to the classical Tikhonov regularization method, has been the object of active research, especially in the case of first order dynamical systems governed by maximal monotone operators, see [24] for some advanced results and references. Concerning second order dynamics, the potential case has been considered in [10]. Let us now state an extension of this last result to the non-potential case.

Corollary 3.1. Let us suppose that $(\mathrm{H} 1)-(\mathrm{H} 3)$ hold with $S:=(\nabla \phi+A)^{-1}(0) \neq \emptyset$ and with $\lambda \gamma^{2}>1$. Let us assume moreover that $t \mapsto \epsilon(t)$ is a decreasing function which converges slowly to zero as $t \rightarrow+\infty$ in the following sense:

$$
\int_{0}^{+\infty} \epsilon(s) \mathrm{d} s=+\infty .
$$

Then, for any $u_{0}$ and $v_{0}$ in $\mathcal{H}$, there exists a unique solution $u \in C^{2}([0,+\infty) ; \mathcal{H})$ of

$$
\ddot{u}(t)+\gamma \dot{u}(t)+\nabla \phi(u(t))+A(u(t))+\epsilon(t) u(t)=0,
$$

with $u(0)=u_{0}$ and $\dot{u}(0)=v_{0}$, which satisfies $u(t) \rightarrow u_{*}$ strongly in $\mathcal{H}$ as $t \rightarrow+\infty$, where $u_{*}$ is the element of minimal norm of the closed convex set $S$.

\section{EXAMPLES}

The following examples aim at illustrating our abstract results. Each of them requires further studies which are out of the scope of the present article.

\subsection{Constrained optimization}

Let $C$ be a closed convex subset of $\mathcal{H}$, and $g: \mathcal{H} \rightarrow \mathbb{R}$ a convex continuously differentiable function, whose gradient $\nabla g$ is Lipschitz continuous over $\mathcal{H}$ with Lipschitz constant $L$. Let us consider the constrained optimization problem

$$
\min \{g(v): v \in C\} .
$$

Because of their direct linking with numerical gradient methods, we are interested in the study of smooth dissipative dynamical systems whose trajectories asymptotically converge towards minimizers of (4.1). Considering second-order dynamics aims at making the algorithms more robust (because of inertia aspects).

Let us denote by $S$ the set of solutions of (4.1) and suppose that $S \neq \emptyset$. A first-order optimality condition for (4.1) is given by the following inclusion

$$
\nabla g(u)+N_{C}(u) \ni 0
$$

where $N_{C}(u)$ is the outward normal cone to $C$ at $u \in C$. Observe that (4.2) is equivalent to

$$
u-P_{C}[u-\mu \nabla g(u)]=0
$$

where $P_{C}$ is the projection operator on $C$ and $\mu$ is a positive parameter. Whence we considerer the following gradient-projection dynamic

$$
\ddot{u}(t)+\gamma \dot{u}(t)+u(t)-P_{C}[u(t)-\mu \nabla g(u(t))]=0 .
$$

This continuous dynamical system has been first introduced by Antipin [8] and further studied by Alvarez and Attouch [4]. An extended study of the corresponding first-order system has been achieved in [18,19]. Let us show that the operator

$$
A v:=v-P_{C}(v-\mu \nabla g(v))
$$


is $(1 / 2)$-cocoercive for $0<\mu<(2 / L)$. To that end, let us notice that $A=I-T$ where $T v=P_{C}(v-\mu \nabla g(v))$. According to Corollary 2.2, and noticing that $P_{C}$ is a contraction, we just need to verify that the mapping $v \mapsto v-\mu \nabla g(v)$ is a contraction too. Given arbitrary $u \in \mathcal{H}, v \in \mathcal{H}$, we have

$$
|(u-\mu \nabla g(u))-(v-\mu \nabla g(v))|^{2}=|u-v|^{2}-2 \mu\langle\nabla g(u)-\nabla g(v), u-v\rangle+\mu^{2}|\nabla g(u)-\nabla g(v)|^{2} .
$$

Thus proving that $v \mapsto v-\mu \nabla g(v)$ is a contraction, is equivalent to prove that $\langle\nabla g(u)-\nabla g(v), u-v\rangle \geq$ $\frac{\mu}{2}|\nabla g(u)-\nabla g(v)|^{2}$, which amounts to say that $\nabla g$ is $(\mu / 2)$-cocoercive. Clearly, this property forces $\nabla g$ to be $(2 / \mu)$-Lipschitz continuous. Indeed, as a striking property, the converse statement holds true for the gradient of a convex function, that's the Baillon-Haddad theorem, see [16]. Hence $\nabla g$ being Lipschitz continuous with Lipschitz constant $L \leq(2 / \mu)$ forces $\nabla g$ to be $(\mu / 2)$-cocoercive.

Hence (4.4) falls in our setting. Applying Theorem 2.1 with $\lambda=1 / 2$, we deduce that, for $\gamma>\sqrt{2}$ and $0<$ $\mu \leq(2 / L)$, every trajectory of (4.4) weakly converges to an element of $S$, which is a minimizer of problem (4.1).

Moreover, when considering the Tikhonov-like regularized dynamic with $\epsilon(t) \rightarrow 0$ as $t$ goes to $+\infty$,

$$
\ddot{u}(t)+\gamma \dot{u}(t)+u(t)-P_{C}[u(t)-\mu \nabla g(u(t))]+\epsilon(t) \nabla \Theta(u(t))=0,
$$

by a direct application of Theorem 3.1, under the assumption $\int_{0}^{+\infty} \epsilon(s) \mathrm{d} s=+\infty$, one obtains that the trajectories of (4.6) strongly converge to the unique solution $\bar{u} \in S$ verifying

$$
\Theta(\bar{u})=\inf _{v \in S} \Theta(v)
$$

Remark 4.1. Inertial dynamical systems, like the "heavy ball with friction dynamical system" have been first introduced by Polyak [34] in the realm of optimization. Since then, an abundant literature has been devoted to this subject. Rich connections between second order dissipative dynamical systems in their respective continuous and discretized forms have been put to the fore, and so provide new algorithms together with a deeper insight into their convergence analysis, see $[2,4-7,10,14,21,26,31]$.

\subsection{Coupled systems and dynamical games}

Throughout this section we make the following standing assumptions:

- $\mathcal{H}=\mathcal{X}_{1} \times \mathcal{X}_{2}$ is the Cartesian product of two Hilbert spaces equipped with norms $|.| \mathcal{X}_{1}$ and $|.| \mathcal{X}_{2}$, while $x=\left(x_{1}, x_{2}\right)$, with $x_{1} \in \mathcal{X}_{1}$ and $x_{2} \in \mathcal{X}_{2}$, stands for an arbitrary element in $\mathcal{H}$;

- $\phi(x)=f_{1}\left(x_{1}\right)+f_{2}\left(x_{2}\right)+\Phi\left(x_{1}, x_{2}\right)$, where $f_{1}: \mathcal{X}_{1} \rightarrow \mathbb{R}, f_{2}: \mathcal{X}_{2} \rightarrow \mathbb{R}$ are smooth convex functions, $\Phi: \mathcal{X}_{1} \times \mathcal{X}_{2} \rightarrow \mathbb{R}$ is a smooth convex coupling function;

- $A=\left(\nabla_{x_{1}} \mathcal{L},-\nabla_{x_{2}} \mathcal{L}\right)$ is the maximal monotone operator which is attached to a smooth convex-concave function $\mathcal{L}: \mathcal{X}_{1} \times \mathcal{X}_{2} \rightarrow \mathbb{R}$. The operator $A$ is assumed to be $\lambda$-cocoercive for some $\lambda>0$.

A typical example of coupling function $\Phi$ (see [15]) is given by

$$
\Phi(x)=\frac{1}{2}\left|L_{1} x_{1}-L_{2} x_{2}\right|_{\mathcal{Z}}^{2}
$$

where $L_{1} \in L\left(\mathcal{X}_{1}, \mathcal{Z}\right)$ and $L_{2} \in L\left(\mathcal{X}_{2}, \mathcal{Z}\right)$ are linear continuous operators acting respectively from $\mathcal{X}_{1}$ and $\mathcal{X}_{2}$ into a third Hilbert space $\mathcal{Z}$ with norm $|\cdot| \mathcal{Z}$.

A smooth convex-concave function $\mathcal{L}$, such that the associated maximal monotone operator $A$ is $\lambda$-cocoercive, can be obtained in a systematic way by using epi-hypo Moreau-Yosida regularization, as described below. Given a general closed convex-concave function $\mathcal{L}: \mathcal{X}_{1} \times \mathcal{X}_{2} \rightarrow \mathbb{R}$ (see [35]) and a positive parameter $\lambda$, we consider $\mathcal{L}_{\lambda}: \mathcal{X}_{1} \times \mathcal{X}_{2} \rightarrow \mathbb{R}$ defined for any $\left(x_{1}, x_{2}\right) \in \mathcal{X}_{1} \times \mathcal{X}_{2}$ by

$$
\mathcal{L}_{\lambda}\left(x_{1}, x_{2}\right)=\min _{u_{1} \in \mathcal{X}_{1}} \max _{u_{2} \in \mathcal{X}_{2}}\left\{\mathcal{L}\left(u_{1}, u_{2}\right)+\frac{1}{2 \lambda}\left|x_{1}-u_{1}\right|_{\mathcal{X}_{1}}^{2}-\frac{1}{2 \lambda}\left|x_{2}-u_{2}\right|_{\mathcal{X}_{2}}^{2}\right\} .
$$


Then $\mathcal{L}_{\lambda}$ is a smooth convex-concave function whose associated maximal monotone operator $\left(\nabla_{x_{1}} \mathcal{L}_{\lambda},-\nabla_{x_{2}} \mathcal{L}_{\lambda}\right)$ is precisely the Yosida approximation $A_{\lambda}$ of the operator $A=\left(\partial_{x_{1}} \mathcal{L},-\partial_{x_{2}} \mathcal{L}\right)$, i.e., $A_{\lambda}=\left(\nabla_{x_{1}} \mathcal{L}_{\lambda},-\nabla_{x_{2}} \mathcal{L}_{\lambda}\right)$ (see [12] for further details).

In this setting, with $u(t)=\left(x_{1}(t), x_{2}(t)\right)$ system (1.3) becomes

$$
\left\{\begin{array}{l}
\ddot{x}_{1}(t)+\gamma \dot{x}_{1}(t)+\nabla f_{1}\left(x_{1}(t)\right)+\nabla_{x_{1}} \Phi\left(x_{1}(t), x_{2}(t)\right)+\nabla_{x_{1}} \mathcal{L}\left(x_{1}(t), x_{2}(t)\right)=0, \\
\ddot{x}_{2}(t)+\gamma \dot{x}_{2}(t)+\nabla f_{2}\left(x_{2}(t)\right)+\nabla_{x_{2}} \Phi\left(x_{1}(t), x_{2}(t)\right)-\nabla_{x_{2}} \mathcal{L}\left(x_{1}(t), x_{2}(t)\right)=0 .
\end{array}\right.
$$

As a straight application of Theorem 2.1, assuming relation $\lambda \gamma^{2}>1$ holds, one obtains $x(t)=\left(x_{1}(t), x_{2}(t)\right) \rightarrow$ $x_{\infty}=\left(x_{1, \infty}, x_{2, \infty}\right)$ weakly in $\mathcal{H}$ as $t$ goes to $+\infty$, where $\left(x_{1, \infty}, x_{2, \infty}\right)$ is solution of the coupled system

$$
\left\{\begin{array}{l}
\nabla f_{1}\left(x_{1}\right)+\nabla_{x_{1}} \Phi\left(x_{1}, x_{2}\right)+\nabla_{x_{1}} \mathcal{L}\left(x_{1}, x_{2}\right)=0 \\
\nabla f_{2}\left(x_{2}\right)+\nabla_{x_{2}} \Phi\left(x_{1}, x_{2}\right)-\nabla_{x_{2}} \mathcal{L}\left(x_{1}, x_{2}\right)=0
\end{array}\right.
$$

Structured systems such as (4.10) contain both potential and non-potential terms whose antagonistic effects are often present in decision sciences and physics.

In game theory, (4.10) describes Nash equilibria of the normal form game with two players 1, 2 whose static loss functions are respectively given by

$$
\left\{\begin{array}{l}
F_{1}:\left(x_{1}, x_{2}\right) \in \mathcal{X}_{1} \times \mathcal{X}_{2} \rightarrow F_{1}\left(x_{1}, x_{2}\right)=f_{1}\left(x_{1}\right)+\Phi\left(x_{1}, x_{2}\right)+\mathcal{L}\left(x_{1}, x_{2}\right), \\
F_{2}:\left(x_{1}, x_{2}\right) \in \mathcal{X}_{1} \times \mathcal{X}_{2} \rightarrow F_{2}\left(x_{1}, x_{2}\right)=f_{2}\left(x_{2}\right)+\Phi\left(x_{1}, x_{2}\right)-\mathcal{L}\left(x_{1}, x_{2}\right),
\end{array}\right.
$$

where the $f_{i}($.$) represent the individual convex payoffs of the players, \Phi(.,$.$) is their joint convex payoff, and \mathcal{L}$ is a convex-concave payoff with zero-sum rule. The case $\mathcal{L}=0$ corresponds to a potential team game (see [32]), while case $\Phi=0$ corresponds to a non cooperative zero-sum game. Game (4.11) involves both cooperative and non cooperative aspects.

A central question in game theory, decision sciences and economics is to describe realistic dynamics which converge to Nash equilibria, see [30] and the references herein. Indeed, implicit time discretization of dynamical system (4.9) leads to the following best response dynamic (players 1 and 2 play alternatively) with inertia and "costs to change"

$$
\left\{\begin{array}{c}
\left(x_{1, k+1}, x_{2, k+1}\right) \rightarrow\left(x_{1, k+2}, x_{2, k+1}\right) \rightarrow\left(x_{1, k+2}, x_{2, k+2}\right) \\
x_{1, k+2}=\operatorname{argmin}_{\xi \in \mathcal{X}_{1}}\left\{f_{1}(\xi)+\Phi\left(\xi, x_{2, k+1}\right)+\mathcal{L}\left(\xi, x_{2, k+1}\right)\right. \\
\left.+\frac{1}{2 \alpha_{k}}\left\|\xi-\left(x_{1, k+1}+\beta_{k}\left(x_{1, k+1}-x_{1, k}\right)\right)\right\|_{\mathcal{X}_{1}}^{2}\right\}, \\
x_{2, k+2}=\operatorname{argmin}_{\eta \in \mathcal{X}_{2}}\left\{f_{2}(\eta)+\Phi\left(x_{1, k+2}, \eta\right)-\mathcal{L}\left(x_{1, k+2}, \eta\right)\right. \\
\left.+\frac{1}{2 \nu_{k}}\left\|\eta-\left(x_{2, k+1}+\beta_{k}\left(x_{2, k+1}-x_{2, k}\right)\right)\right\|_{\mathcal{X}_{2}}^{2}\right\} .
\end{array}\right.
$$

The terms $\left\|\xi-x_{1, k+1}-\beta_{k}\left(x_{1, k+1}-x_{1, k}\right)\right\|^{2}$ and $\left\|\eta-x_{2, k+1}-\beta_{k}\left(x_{2, k+1}-x_{2, k}\right)\right\|^{2}$ naturally come into play after discretizing first and second order time derivatives, see [2,5]. In decision sciences, they reflect some aspects of agents behaviors like anchoring and inertia, and can be interpreted as "low local costs to change", see [11,15]. The various parameters $\alpha_{k}, \nu_{k}, \beta_{k}$ model adaptive, learning abilities of the agents as well as their reactivity or resistance to change.

Continuous dynamical system (4.9) together with its convergence properties offers a valuable guideline to study discrete dynamic (4.12). Clearly, this requires further study beyond the limit of the present paper.

\subsection{Further perspectives}

It is natural to relax the regularity assumptions on the potential operator $\nabla \phi$ and the maximal monotone operator $A$, and to wonder whether our asymptotic convergence results remain valid in respect of the more 
general differential inclusion

$$
\ddot{u}(t)+\gamma \dot{u}(t)+A u(t)+\partial \phi(u(t)) \ni 0 .
$$

Let us give some precisions (regarding cases when either $A$ or $\partial \phi$ is single-valued):

1. The non-smooth potential case (obtained by taking $A=0$ ), that is

$$
\ddot{u}(t)+\gamma \dot{u}(t)+\partial \phi(u(t)) \ni 0,
$$

has been considered in the finite dimensional case in [14]. Trajectories of (4.14) naturally exhibit elastic shocks, which makes this system play an important role in unilateral mechanics, see [36]. Taking $\phi$ equal to the indicator function of a closed convex set $C$ yields (damped) billiard dynamics. Given a non-smooth closed convex potential $\phi: \mathcal{H} \rightarrow \mathbb{R} \cup\{+\infty\}$ and a $\lambda$-cocoercive operator $A$, a natural approach to (4.13) (in the same lines as [14]) would consist in approximating $\phi$ by smooth convex functions $\phi_{n}$ (this is always possible for example via Moreau-Yosida approximation), which leads to study

$$
\ddot{u}_{n}(t)+\gamma \dot{u}_{n}(t)+A u_{n}(t)+\nabla \phi_{n}\left(u_{n}(t)\right)=0 .
$$

Note that our basic assumption $\lambda \gamma^{2}>1$, which does not concern the potential part of the operator, is not affected by this operation. Thus, (4.15) falls in our setting. Then, one has to establish estimations on the sequence $\left(u_{n}\right)$, pass to the limit on these estimations as $n$ goes to infinity, and derive conclusions on the asymptotic behavior of trajectories of (4.13). As it has been done in [6,14], this program would allow to consider the asymptotic behavior of damped hyperbolic equations (with non-isolated equilibria) combining potential with non potential effects.

2. The study of (4.13) with a general maximal monotone operator $A$ leads to considerable difficulties. In that case, another type of regularization or relaxation method can be used. It relies on the following remark. Take $\lambda>0$ and $\gamma>0$ such that $\lambda \gamma^{2}>1$. Then consider the equation

$$
\ddot{u}(t)+\gamma \dot{u}(t)+\nabla \phi(u(t))+A_{\lambda}(u(t))=0,
$$

where $A_{\lambda}$ is the Yosida approximation of $A$. Equation (4.16) enters our setting (as $A_{\lambda}$ is $\lambda$-cocoercive) and it can be equivalently rewritten as

$$
\left\{\begin{array}{l}
\ddot{u}(t)+\gamma \dot{u}(t)+\nabla \phi(u(t))+\frac{1}{\lambda}(u(t)-v(t))=0, \\
A v(t)+\frac{1}{\lambda}(v(t)-u(t)) \ni 0 .
\end{array}\right.
$$

This naturally suggests that some of our results can be extended to coupled systems of the following type

$$
\left\{\begin{array}{l}
\ddot{u}(t)+\gamma \dot{u}(t)+\nabla \phi(u(t))+\frac{1}{\lambda}(u(t)-v(t))=0, \\
\dot{v}(t)+A v(t)+\frac{1}{\lambda}(v(t)-u(t)) \ni 0 .
\end{array}\right.
$$

Our approach, which is based on Lyapunov methods [22,28,29], can be adapted to such nonlinear systems. In the case of linear hyperbolic PDE's, it would be worthwhile to compare it with spectral analysis methods, see for example $[25,27]$.

3. In view of further applications, it would be interesting to consider other types of dissipation phenomena: For example, dry friction most likely produces stabilization within finite time, a desirable feature of human decision processes, see [1]. Geometrical damping is of great importance for numerical optimization, and modeling of non elastic shocks in mechanics, see [7].

Acknowledgements. The authors are grateful to the anonymous referee who has provided us with the nice Example 1 in Section 2.4. 


\section{REFERENCES}

[1] S. Adly, H. Attouch and A. Cabot, Finite time stabilization of nonlinear oscillators subject to dry friction - Nonsmooth mechanics and analysis. Adv. Mech. Math. 12 (2006) 289-304.

[2] F. Alvarez, On the minimizing property of a second order dissipative system in Hilbert space. SIAM J. Control Optim. 38 (2000) 1102-1119.

[3] F. Alvarez, Weak convergence of a relaxed and inertial hybrid projection-proximal point algorithm for maximal monotone operators in Hilbert space. SIAM J. Optim. 14 (2004) 773-782.

[4] F. Alvarez and H. Attouch, The heavy ball with friction dynamical system for convex constrained minimization problems, in Optimization, Namur (1998), Lecture Notes in Econom. Math. Systems 481, Springer, Berlin (2000) 25-35.

[5] F. Alvarez and H. Attouch, An inertial proximal method for monotone operators via discretization of a nonlinear oscillator with damping. Set Valued Anal. 9 (2001) 3-11.

[6] F. Alvarez and $\mathrm{H}$. Attouch, Convergence and asymptotic stabilization for some damped hyperbolic equations with non-isolated equilibria. ESAIM: COCV 6 (2001) 539-552.

[7] F. Alvarez, H. Attouch, J. Bolte and P. Redont, A second-order gradient-like dissipative dynamical system with Hessian-driven damping. Application to optimization and mechanics. J. Math. Pures Appl. 81 (2002) 747-779.

[8] A.S. Antipin, Minimization of convex functions on convex sets by means of differential equations. Differ. Uravn. 30 (1994) 1475-1486 (in Russian). English translation: Diff. Equ. 30 (1994) 1365-1375.

[9] H. Attouch and R. Cominetti, A dynamical approach to convex minimization coupling approximation with the steepest descent method. J. Diff. Equ. 128 (1996) 519-540.

[10] H. Attouch and M.-O. Czarnecki, Asymptotic control and stabilization of nonlinear oscillators with non-isolated equilibria. J. Diff. Equ. 179 (2002) 278-310.

[11] H. Attouch and A. Soubeyran, Inertia and reactivity in decision making as cognitive variational inequalities. J. Convex. Anal. 13 (2006) 207-224.

[12] H. Attouch, D. Aze and R. Wets, Convergence of convex-concave saddle functions: Applications to convex programming and mechanics. Ann. Inst. Henri Poincaré, Anal. Non Linéaire 5 (1988) 537-572.

[13] H. Attouch, X. Goudou and P. Redont, The heavy ball with friction method: The continuous dynamical system. Global exploration of local minima by asymptotic analysis of a dissipative dynamical system. Commun. Contemp. Math. 1 (2000) $1-34$.

[14] H. Attouch, A. Cabot and P. Redont, The dynamics of elastic shocks via epigraphical regularization of a differential inclusion. Barrier and penalty approximations. Adv. Math. Sci. Appl. 12 (2002) 273-306.

[15] H. Attouch, J. Bolte, P. Redont and A. Soubeyran, Alternating proximal algorithms for weakly coupled minimization problems. Applications to dynamical games and PDE's. J. Convex Anal. 15 (2008) 485-506.

[16] J.-B. Baillon and G. Haddad, Quelques propriétés des opérateurs angles-bornés et n-cycliquement monotones. Israel J. Math. 26 (1977) 137-150.

[17] J.-B. Baillon and A. Haraux, Comportement à l'infini pour les équations d'évolution avec forcing périodique. Arch. Rat. Mech. Anal. 67 (1977) 101-109.

[18] J. Bolte, Continuous gradient projection method in Hilbert spaces. J. Optim. Theory Appl. 119 (2003) 235-259.

[19] J. Bolte and M. Teboulle, Barrier operators and associated gradient-like dynamical systems for constrained minimization problems. SIAM J. Control Optim. 42 (2003) 1266-1292.

[20] H. Brézis, Opérateurs maximaux monotones et semi-groupes de contractions dans les espaces de Hilbert, Mathematical Studies. North-Holland (1973).

[21] A. Cabot, Inertial gradient-like dynamical system controlled by a stabilizing term. J. Optim. Theory Appl. 120 (2004) $275-303$.

[22] T. Cazenave and A. Haraux, An introduction to semilinear evolution equations, Oxford Lecture Series in Mathematics and its Applications 13. Oxford University Press, Oxford (1998).

[23] P.L. Combettes and S.A. Hirstoaga, Visco-penalization of the sum of two operators. Nonlinear Anal. 69 (2008) 579-591.

[24] R. Cominetti, J. Peypouquet and S. Sorin, Strong asymptotic convergence of evolution equations governed by maximal monotone operators with Tikhonov regularization. J. Diff. Equ. 245 (2008) 3753-3763.

[25] S. Ervedoza and E. Zuazua, Uniformly exponentially stable approximations for a class of damped systems. J. Math. Pures Appl. 91 (2009) 20-48.

[26] S.D. Flam and J. Morgan, Newtonian mechanics and Nash play. Int. Game Theory Rev. 6 (2004) 181-194.

[27] I. Gallagher, Asymptotics of the solutions of hyperbolic equations with a skew-symmetric perturbation. J. Diff. Equ. 150 (1998) 363-384.

[28] J.K. Hale and G. Raugel, Convergence in gradient-like systems with applications to PDE. Z. Angew. Math. Phys. 43 (1992) $63-125$.

[29] A. Haraux, Systèmes dynamiques dissipatifs et applications 17. Masson, RMA (1991).

[30] J. Hofbauer and S. Sorin, Best response dynamics for continuous zero-sum games. Discrete Continuous Dyn. Syst. Ser. B 6 (2006) 215-224. 
[31] P.E. Maingé, Regularized and inertial algorithms for common fixed points of nonlinear operators. J. Math. Anal. Appl. 344 (2008) 876-887.

[32] D. Monderer and L.S. Shapley, Potential Games. Games Econ. Behav. 14 (1996) 124-143.

[33] Z. Opial, Weak convergence of the sequence of successive approximations for nonexpansive mappings. Bull. Amer. Math. Soc. 73 (1967) 591-597.

[34] B.T. Polyak, Introduction to Optimization. Optimization Software, New York (1987).

[35] R.T. Rockafellar, Monotone operators associated with saddle-functions and mini-max problems, in Nonlinear operators and nonlinear equations of evolution in Banach spaces 2, 18th Proceedings of Symposia in Pure Mathematics, F.E. Browder Ed., American Mathematical Society (1976) 241-250.

[36] M. Schatzman, A class of nonlinear differential equations of second order in time. Nonlinear Anal. 2 (1978) 355-373. 